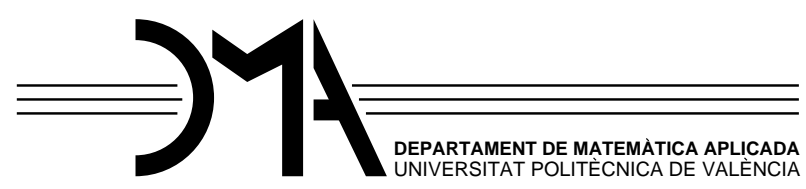

\title{
Subgrupos solitarios de grupos finitos
}

Orieta Liriano Castro

Trabajo de tesis doctoral presentado por Orieta Liriano Castro para optar al título de Doctora en Matemáticas Multidisciplinares por la Universitat Politècnica de València bajo la dirección del Dr. Ramón Esteban Romero

Valencia, 14 de diciembre de 2015 



\section{Índice general}

$\begin{array}{ll}\text { Resumen } & 7\end{array}$

$\begin{array}{ll}\text { Resum } & 9\end{array}$

$\begin{array}{ll}\text { Summary } & 11\end{array}$

$\begin{array}{ll}\text { Agradecimientos } & 13\end{array}$

1. Preliminares 15

1.1. Grupos resolubles . . . . . . . . . . . . . . . . . 15

1.2. Grupos superresolubles y nilpotentes . . . . . . . . . . 18

1.3. Clases de grupos . . . . . . . . . . . . . . . 20

1.4. Grupos $p$-resolubles y $p$-nilpotentes . . . . . . . . . 22

2. Definiciones y propiedades básicas 25

2.1. Subgrupos solitarios y subgrupos normales solitarios . . 25

2.2. Subgrupos solitarios para cocientes . . . . . . . . 27

3. Propiedades reticulares $\quad 31$

3.1. Observaciones sobre el retículo de subgrupos solitarios . 31

4. Subgrupos solitarios y clases de grupos 37

4.1. Subgrupos solitarios de tipo radical y residual . . . . . . 37

4.2. Radicales solitarios para cocientes y residuales solitarios 40

4.3. Subgrupos solitarios no radicales . . . . . . . . . . 42

5. Grupos cuyos subgrupos minimales son solitarios 47

5.1. Grupos cuaternios generalizados . . . . . . . . . . . . 4 47

5.2. Enunciado del teorema . . . . . . . . . . . . . . . . . 49 
5.3. Demostración de los resultados . . . . . . . . . . . . 50

5.4. Observaciones finales . . . . . . . . . . . . . 57

Bibliografía 


\section{Índice de figuras}

3.1. Retículo de subgrupos solitarios para cocientes del grupo del ejemplo $3.4 \ldots \ldots \ldots$. . . . . . . . . . . 33

3.2. Conjunto parcialmente ordenado de los subgrupos normales solitarios del grupo del ejemplo 3.5 . . . . . . . . 34

3.3. Retículo de subgrupos subnormales solitarios del grupo de los ejemplos 3.5 y $3.11 \ldots \ldots$. . . . . . . . . 36 



\section{Resumen}

El ámbito de esta tesis es el de la teoría abstracta de grupos finitos. Todos los grupos que consideremos serán finitos. Por ello, la palabra «grupo» se entenderá como sinónima de «grupo finito». Decimos que un subgrupo $H$ de un grupo $G$ es solitario cuando ningún otro subgrupo de $G$ es isomorfo a $H$. Un subgrupo normal $H$ de un grupo $G$ se dice normal solitario cuando ningún otro subgrupo normal de $G$ es isomorfo a $H$. Un subgrupo normal $N$ de un grupo $G$ se dice que es solitario para cocientes cuando ningún otro subgrupo normal $K$ de $G$ da un cociente isomorfo a $G / N$. Los subgrupos solitarios, los subgrupos normales solitarios y los subgrupos solitarios para cocientes han sido recientemente estudiados por autores como Thévenaz [Thé93], quien bautizó los subgrupos solitarios como subgrupos fuertemente característicos, Kaplan y Levy [KL09, Lev14], Tărnăuceanu [Tăr12b, Tăr12a] y Atanasov y Foguel [AF12].

El objeto de este proyecto de tesis doctoral es el de profundizar en el análisis de estas propiedades de inmersión de subgrupos, afinando en el conocimiento de sus propiedades reticulares, obteniendo propiedades generales en relación con clases de grupos y analizando grupos en los que los miembros de algunas familias destacadas de subgrupos satisfacen estas propiedades de inmersión.

Los resultados básicos de teoría de grupos que se utilizan en la memoria aparecen en el capítulo 1. Entre ellos, comentamos algunos resultados sobre grupos resolubles, superresolubles, nilpotentes, clases de grupos y grupos $p$-resolubles y $p$-nilpotentes para un primo $p$. En el capítulo 2 presentamos los conceptos básicos sobre estas propiedades de inmersión, así como algunos resultados básicos que satisfacen.

El capítulo 3 está dedicado al estudio de propiedades reticulares de estos tipos de subgrupos. En este capítulo se profundiza en el estudio de los retículos de subgrupos solitarios y solitarios para cocientes lle- 
vado a cabo por Kaplan y Levy [KL09] y por Tărnăuceanu [Tăr12b] y se comprueba que, a pesar de que estos retículos constan de subgrupos normales, no son subretículos del retículo de subgrupos normales. También comprobamos que el conjunto de subgrupos normales solitarios no constituye un retículo, lo que motiva la introducción del concepto de subgrupo subnormal solitario como herramienta más adecuada para tratar propiedades reticulares.

En el capítulo 4 estudiamos con profundidad las relaciones entre estas propiedades de inmersión y clases de grupos. Observamos que los subgrupos subnormales solitarios se comportan bien respecto de radicales de clases de Fitting y que los residuales para formaciones son subgrupos solitarios para cocientes. Esto permite mejorar algunos resultados sobre subgrupos solitarios para cocientes. También estudiamos condiciones en que los radicales respecto de clases de Fitting son subgrupos solitarios para cocientes y los residuales respecto de formaciones son subgrupos solitarios. Por último, nos planteamos la cuestión natural de si los subgrupos solitarios o subnormales solitarios pueden verse como radicales para clases de Fitting adecuadas o si los subgrupos solitarios para cocientes son residuales para clases de Fitting adecuadas. Damos una respuesta negativa a esta cuestión.

El capítulo 5 está dedicado al estudio de grupos cuyos subgrupos minimales son solitarios, es decir, grupos con un único subgrupo de orden $p$ para cada primo $p$ divisor de su orden. Damos una clasificación completa de estos grupos y hacemos algunas observaciones sobre problemas relacionados.

Nuestras aportaciones a esta línea de investigación aparecen en el artículo [ERL], aceptado para su publicación en Communications in Algebra, cuyos resultados aparecen principalmente en los capítulos 3 y 4 , y en [ERL16], aceptado para su publicación en Journal of Algebra and its Applications, cuyos resultados aparecen principalmente en el capítulo 5. También han sido presentados en el IX Encuentro en Teoría de Grupos [ERL12], en el Seminario Predoc de la Universitat de València [LC13] y en el X Congreso Internacional de Investigación Científica organizado por la Universidad Autónoma de Santo Domingo [ERL14]. 


\section{Resum}

L'àmbit d'aquesta tesi és el de la teoria abstracta de grups finits. Tots els grups que hi considerem seran finits. Per això, la paraula "grup» s'entendrà com a sinònima de «grup finit». Direm que un subgrupo $H$ d'un grup $G$ és solitari quan cap altre subgrup de $G$ no és isomorf a $H$. Un subgrup normal $H$ d'un grup $G$ es diu normal solitari quan cap altre subgrup normal de $G$ no és isomorf a $H$. Un subgrup normal $N$ d'un grup $G$ es diu que és solitari per a quocients quan cap altre subgrup normal $K$ de $G$ no dóna un quocient isomorf a $G / N$. Els subgrups solitaris, els subgrups solitaris normals i els subgrups solitaris per a quocients han sigut recentment estudiats per autors com Thévenaz [Thé93], qui batejà els subgrups solitaris com a subgrups fortament característics, Kaplan i Levy [KL09, Lev14], Tărnăuceanu [Tăr12b, Tăr12a] i Atanasov i Foguel [AF12].

L'objecte d'aquest projecte de tesi doctoral és el d'aprofundir en l'anàlisi d'aquestes propietats d'immersió de subgrups, afinant en el coneixement de les seues propietats reticulars, obtenint propietats generals en relació amb classes de grups i analitzant grups en què els membres d'algunes famílies destacades de subgrups satisfan aquestes propietats d'immersió.

Els resultats bàsics de teoria de grups que es fan servir en la memòria apareixen al capítol 1. Entre ells, comentem alguns resultats sobre grups resolubles, superresolubles, nilpotents, classes de grups i grups $p$-resolubles i $p$-nilpotents per a un primer $p$. Al capítol 2 presentem els conceptes bàsics sobre aquestes propietats d'immersió, així com alguns resultats bàsics que satisfan.

El capítol 3 està dedicat a l'estudi de propietats reticulars d'aquests tipus de subgrups. En aquest capítol s'aprofundeix en l'estudi dels reticles de subgrups solitaris i solitaris per a quocients dut a terme per Kaplan i Levy [KL09] i per Tărnăuceanu [Tăr12b] i es comprova que, 
encara que aquests subgrups consten de subgrups normals, no són subreticles del reticle de subgrups normals. També comprovem que el conjunt de subgrups normals solitaris no constitueix un reticle, la qual cosa motiva la introducció del concepte de subgrup subnormal solitari com a eina més adient per tractar propietats reticulars.

Al capítol 4 estudiem amb profunditat les relacions entre aquestes propietats d'immersió i classes de grups. Observem que els subgrups subnormals solitaris es comporten bé respecte de radicals de classes de Fitting i que els residuals per a formacions són subgrups solitaris per a quocients. Açò permet millorar alguns resultats sobre subgrups solitaris per a quocients. També estudien condicions en què els radicals respecte de classes de Fitting són subgrups solitaris per a quocients i els residuals respecte de formacions són subgrups solitaris. Per acabar, ens plantegem la qüestió natural de si els subgrups solitaris o subnormals solitaris poden veure's com a radicals per a classes de Fitting adients o si els subgrups solitaris per a quocients són residuals per a classes de Fitting adients. Donem una resposta negativa a aquesta qüestió.

El capítol 5 està dedicat a l'estudi de grups els subgrups minimals dels quals són solitaris, és a dir, grups amb un únic subgrup d'ordre $p$ per a cada primer $p$ divisor del seu ordre. Donem una classificació completa d'aquests grups i fem algunes observacions sobre problemes relacionats.

Les nostres aportacions a aquesta línia de recerca apareixen en l'article [ERL], acceptat per a la seua publicació a Communications in Algebra, els resultats del qual apareixen principalment als capítols 3 i 4, i en [ERL16], acceptat per a la seua possible publicació a Journal of Algebra and its Applications, els resultats del qual apareixen principalmente al capítol 5. També han sigut presentats al congrés IX Encuentro en Teoría de Grupos [ERL12], al Seminari Predoc de la Universitat de València [LC13] i al X Congreso Internacional de Investigación Científica organitzat per la Universidad Autónoma de Santo Domingo [ERL14]. 


\section{Summary}

The scope of this thesis is the abstract finite group theory. All the groups we will consider will be finite. hence, the word "group" will be understood as a synonimous of "finite group". We say that a subgroup $H$ of a group $G$ is solitary when no other subgroup of $G$ is isomorphic to $H$. A normal subgroup $H$ of a group $G$ is said to be normal solitary when no other normal subgroup of $G$ is isomorphic to $H$. A normal subgroup $N$ of a group $G$ is said to be quotient solitary when no other normal subgroup $K$ of $G$ gives a quotient isomorphic to $G / N$. Solitary subgroups, normal solitary subgroups, and quotient solitary subgroups have been recently studied by authors like Thévenaz [Thé93], who named the solitary subgroups as strongly characteristic subgroups, Kaplan and Levy [KL09, Lev14], Tărnăuceanu [Tăr12b, Tăr12a], and Atanasov and Foguel [AF12].

The aim of this PhD thesis project is to deepen into the analysis of these subgroup embedding properties, by refining the knowledge of their lattice properties, by obtaining general properties related to classes of groups, and by analysing groups in which the members of some distinguished families of subgroups satisfy these embedding properties.

The basic results of group theory that will be used in the memoir appear in Chapter 1. Among them, we comment on some results about soluble groups, supersoluble groups, nilpotent groups, classes of groups, and $p$-soluble and $p$-nilpotent groups for a prime $p$. In Chapter 2, we present the basic concepts about these embedding properties, as well as some basic results satisfied by them.

Chapter 3 is devoted to the study of lattice properties of these types of subgroups. In this chapter we deepen into the study of the lattices of solitary subgroups and quotient solitary subgroups developed by Kaplan and Levy [KL09] and by Tărnăuceanu [Tăr12b] and we check 
that, even though these lattices consist of normal subgroups, they are not sublattices of the lattice of normal subgroups. We also check that the set of all normal solitary subgroups does not constitute a lattice, which motivates the introduction of the concept of subnormal solitary subgroup as a more suitable tool to deal with lattice properties.

In Chapter 4, we study in depth the relations between these embedding properties and classes of groups. We observe that the subnormal solitary subgroups behave well with respect to radicals for Fitting classes and that the residuals for formations are quotient solitary subgroups. We also study conditions under which the radicals with respect to Fitting classes are quotient solitary subgroups and the residuals with respect to formations are solitary subgroups. To finish, we state the natural question of whether the solitary or subnormal solitary subgroups can be regarded as radicals for suitable Fitting classes or whether the quotient solitary subgroups are residuals for suitable Fitting classes. We give a negative answer to this question.

Chapter 5 is devoted to the study of groups whose minimal subgroups are solitary, that is, groups with a unique subgroup of order $p$ for each prime $p$ dividing its order. We give a complete classification of these groups and we make some remarks about related problems.

Our contributions to this research line appear in the paper [ERL], accepted to be published in Communications in Algebra, whose results appear mainly in Chapters 3 and 4, and in [ERL16], accepted to be published in Journal of Algebra and its Applications, whose results appear mainly in Chapter 5. They have been also presented in the IX Encuentro en Teoría de Grupos (Spanish Meeting in Group Theory) [ERL12], in the Predoc Seminar of the Universitat de València [LC13] and in the $X$ Congreso Internacional de Investigación Cientifica (X International Conference of Scientific Research) organised by the Universidad Autónoma de Santo Domingo [ERL14]. 


\section{Agradecimientos}

- A Dios, guía en cada paso de mi existencia,

- al Dr. Ramón Esteban Romero por su extraordinaria calidad humana y convertirse en el andamiaje perfecto para el logro de este proyecto,

- al Dr. Adolfo Ballester Bolinches por su visión y soporte en la elección de esta investigación,

- a los Dres. Gil Kaplan y Jack Schmidt por sus conversaciones y aportes útiles sobre este tema,

- a los evaluadores externos, por el trabajo de revisión y los comentarios sobre la tesis,

- a la Universidad Politécnica de Valencia y a la Universidad de Valencia por el uso de sus infraestructuras físicas y la eficiencia de su personal de servicio, y especialmente a la primera universidad por el soporte financiero en algunas de nuestras visitas,

- a la Universidad Iberoamericana y a la Universidad Autónoma de Santo Domingo por el soporte financiero y apoyo institucional durante las diferentes etapas,

- al Ministerio de Ciencia e Innovación y al Ministerio de Economía y Competitividad de España por el soporte financiero a través de los proyectos MTM2010-19038-C03-01 y MTM2014-54707-C3-1-P, respectivamente,

- a los compañeros de mi tierra, República Dominicana, y de España, por sus estímulos motivadores, 
- a mi familia: en especial a José Luis, José Manuel y José Emilio, por sus compañías y amor incondicional:

Gracias. 


\section{Capítulo 1}

\section{Preliminares}

En este capítulo presentamos algunos resultados básicos de teoría de grupos que serán utilizados a lo largo de esta memoria. La notación que vamos a usar es la estándar en la teoría de grupos y se corresponde con la que se puede encontrar en libros como el de Doerk y Hawkes [DH92], Huppert [Hup67] o Gorenstein [Gor80]. El lector también podrá hallar en estos libros los resultados básicos sobre teoría de grupos finitos. Todos los grupos con los que vamos a tratar en esta memoria van a ser finitos. Por lo tanto, en lo sucesivo, la palabra «grupo» se entenderá como sinónima de «grupo finito».

\subsection{Grupos resolubles}

Definición 1.1. Diremos que un grupo es resoluble si existe $n \geqslant 0$ tal que $G^{(n)}=1$, donde $G^{(n)}$ es el $n$-ésimo término de la serie derivada de $G$.

Así si $G$ es resoluble, $G$ tiene una serie

$$
1=A_{0} \preccurlyeq A_{1} \preccurlyeq A_{2} \ldots \preccurlyeq A_{r}=G
$$

en la que cada grupo cociente $A_{i-1} / A_{i}$, es abeliano para todo $i$.

Los grupos resolubles presentan propiedades interesantes:

Proposición 1.2 ([DH92, Chapter A, Theorem 10.2]). Sea G un grupo, $H \leqslant G$ y $M, N \leqslant G$. Entonces:

1. Si Ges resoluble, entonces $H$ es resoluble. 
2. Ges resoluble si, y solo si, $G / N$ es resoluble y $N$ es resoluble.

3. Si $G / M$ y $G / N$ son resolubles, entonces $G /(M \cap N)$ es resoluble.

4. Si M y N son resolubles, entonces $M N$ es resoluble.

Denotamos el conjunto de todos los números naturales primos por $\mathbb{P}$. Si $\pi \subseteq \mathbb{P}$, denotamos por $\pi^{\prime}=\mathbb{P} \backslash \pi$. Si $p$ es un primo, denotamos por $p^{\prime}=\mathbb{P} \backslash\{p\}$.

Definiciones 1.3 ([DH92, Chapter I, Definitions 3.1]). Sea $\pi$ un conjunto de números primos.

1. Un $\pi$-número es un número entero cuyos divisores primos pertenecen todos a $\pi$.

2. Un subgrupo $H$ de un grupo $G$ se dice que es un $\pi$-subgrupo de Hall si $|H|$ es un $\pi$-número y $|G: H|$ es un $\pi^{\prime}$-número.

3. Un subgrupo $H$ de $G$ se dice que es subgrupo de Hall si es un $\pi$-subgrupo de Hall de $G$ para algún $\pi \subseteq \mathbb{P}$. Evidentemente, $H$ es un subgrupo de Hall de $G$ si, y solo si, $\operatorname{mcd}(|G: H|,|H|)=1$.

Las propiedades fundamentales de los subgrupos de Hall pueden verse en [DH92, Chapter I, Section 3]. Los subgrupos de Hall generalizan los subgrupos de Sylow a conjuntos de primos, ya que un $p$-subgrupo de Sylow es un $\{p\}$-subgrupo de Hall. Sin embargo, mientras la existencia de $p$-subgrupos de Sylow está garantizada para todo primo $p$ divisor del orden del grupo en virtud de los teoremas de Sylow, no siempre existen $\pi$-subgrupos de Hall para un conjunto de primos $\pi$. Por ejemplo, el grupo alternado $A_{5}$ de grado 5 no posee $\{3,5\}$-subgrupos de Hall. De hecho, la existencia de $\pi$-subgrupos de Hall para todo conjunto de primos $\pi$ es una propiedad característica de los grupos resolubles, como muestran los siguientes resultados de Hall.

Teorema 1.4 (Hall [Hal28], véase también [DH92, Chapter I, Theorem 3.3]). Sea G un grupo resoluble y $\pi$ un conjunto de primos, entonces:

1. G posee $\pi$-subgrupos de Hall,

2. los $\pi$-subgrupos de Hall de G forman una clase de conjugación de G,y 
3. cada $\pi$-subgrupo de G está contenido en un $\pi$-subgrupo de Hall de G.

Teorema 1.5 (Hall [Hal37a], véase también [DH92, Chapter I, Theorem 3.5]). Sea $G$ un grupo con $|G|=\prod_{j=1}^{r} p_{j}^{a_{j}}$, donde $p_{1}, \ldots, p_{r}$ son primos distintos dos a dos. Supongamos que $G$ posee subgrupos $S_{1}, \ldots, S_{r}$ tales que $\left|G: S_{i}\right|=p_{i}^{a_{i}}$ para $1 \leqslant i \leqslant r$, en otras palabras, que $G$ posee $p^{\prime}$-subgrupos de Hall para todo $p \in \mathbb{P}$. Entonces $G$ es resoluble.

Como consecuencia de los teoremas 1.4 y 1.5, se tiene el siguiente resultado fundamental.

Teorema 1.6. Un grupo $G$ es resoluble si, y solo si, $G$ posee $\pi$-subgrupos de Hall para todos los conjuntos $\pi$ de primos.

Como vemos en el teorema 1.5, la existencia de $p^{\prime}$-subgrupos de Hall para cada primo $p$ es una propiedad característica de los grupos finitos resolubles. También lo es la existencia de sistemas de Hall, introducidos en [Hal37b]. Seguiremos el planteamiento más general presentado por Doerk y Hawkes en [DH92, Chapter I, Section 4], que es más adecuado para nuestros intereses.

Definición 1.7. Sea $G$ un grupo resoluble y denotemos por $\pi(G)$ el conjunto de divisores primos de $|G|$. Un sistema de Hall de $G$ es un conjunto $\mathbb{H}$ de subgrupos de Hall de $G$ que satisfacen las dos propiedades siguientes:

1. Para cada $\pi \subseteq \pi(G), \mathbb{H}$ contiene exactamente un $\pi$-subgrupo de Hall $G$, que denotamos por $G_{\pi}$.

2. Si $H, K \in \mathbb{H}$, entonces $H K=K H$.

Si $\mathbb{H}$ es un sistema de Hall de $G$ y, para cada $\pi \subseteq \pi(G), G_{\pi}$ denota el único $\pi$-subgrupo de Hall de $G$ en $\mathbb{H}$, entonces la aplicación $\pi \longmapsto G_{\pi}$, $\pi \subseteq \pi(G)$, del conjunto potencia de $\pi(G)$ en $\mathbb{H}$ es biyectiva y conserva el orden parcial dado por la inclusión ([DH92, Chapter I, Lemma 4.2]).

Proposición 1.8 ([DH92, Chapter I, Proposition 4.4]). Sea G un grupo resoluble y sea $\pi(G)=\left\{p_{1}, \ldots, p_{r}\right\}$. Para cada $i \in\{1, \ldots, r\}$, sea $S_{i}$ un $p_{i}^{\prime}$ subgrupo de Hall de $G$ y sea $\mathbb{K}=\left\{G, S_{1}, \ldots, S_{r}\right\}$. Si $\pi \subseteq \pi(G)$, sea $\pi^{*}=$ $\pi(G) \backslash \pi$ y sea $G_{\pi}=\bigcap\left\{S_{i} \mid p_{i} \in \pi^{*}\right\}$. Entonces

1. $\mathbb{H}=\left\{G_{\pi} \mid \pi \subseteq \pi(G)\right\}$ es un sistema de Hall de $G, y$

2. $\mathbb{H}$ es el único sistema de Hall de G en que $\mathbb{K}$ está contenido. 


\subsection{Grupos superresolubles y nilpotentes}

Las propiedades principales de los grupos superresolubles aparecen en [Hup67, Kapitel VI, §9]. Recordamos la definición de esta propiedad de grupos.

Definición 1.9. Un grupo G es superresoluble si posee una serie normal finita,

$$
1=A_{0} \Downarrow A_{1} \Downarrow A_{2} \Downarrow \cdots \Downarrow A_{r}=G
$$

en la que cada grupo cociente $A_{i} / A_{i-1}$, es cíclico para cada $i \in\{1, \ldots, r\}$.

Teorema 1.10 (véase [Hup67, Kapitel VI, Satz 9.1 y notas previas]). Supongamos que G es un grupo superresoluble.

1. Si $G$ es superresoluble y $H \leqslant G$, entonces $H$ es superresoluble.

2. Si G es superresoluble y $N \leqslant G$, entonces $G / N$ es superresoluble.

3. Si $N_{1}, N_{2} \Downarrow G$ y los cocientes $G / N_{1}$ y $G / N_{2}$ son superresolubles, entonces $G /\left(N_{1} \cap N_{2}\right)$ es superresoluble.

4. Si $G$ es un grupo superresoluble y $p$ es el mayor primo divisor de $|G|$, entonces $G$ tiene un p-subgrupo de Sylow normal. En particular, G posee una torre de Sylow para el orden natural de los números primos divisores de $|G|$, es decir, si los primos divisores de $|G|$ son $p_{1}<p_{2}<\cdots<p_{r}$, existe una cadena de subgrupos

$$
1=P_{r} \leqslant P_{r-1} \leqslant P_{r-2} \leqslant \cdots \leqslant P_{1} \leqslant P_{0}=G
$$

de manera que $P_{k}$ es normal en $G$ para $1 \leqslant k \leqslant r$ y $P_{k-1} / P_{k}$ es un $p_{k}$-subgrupo de Sylow normal de $G / P_{k}$ para $1 \leqslant k \leqslant r$.

Las propiedades fundamentales de los grupos nilpotentes se pueden encontrar en [DH92, Chapter A, Section 8].

Definición 1.11. La serie central descendente de un grupo $G$ viene definida por $K_{1}(G)=G, K_{n}(G)=\left[K_{n-1}(G), G\right]$ para $n \geqslant 2$.

Definición 1.12. Un grupo $G$ es nilpotente cuando existe un natural $c$ de manera que el término $(c+1)$-ésimo $K_{c+1}(G)$ de la serie central descendente de $G$ es trivial. 
Definición 1.13. Si $G$ es un grupo nilpotente, decimos que $G$ es de clase de nilpotencia 0 si $G=1$ y de clase de nilpotencia $c=c(G)$ si la serie central descendente de $G$ satisface $K_{c}(G) \neq 1=K_{c+1}(G)$.

Claramente, los grupos nilpotentes son superresolubles. El siguiente resultado expresa algunas propiedades de grupos nilpotentes.

Proposición 1.14. 1. Los subgrupos e imágenes homomorfas de grupos nilpotentes son nilpotentes.

2. El producto directo de grupos nilpotentes es nilpotente.

3. Para cada primo p, los p-grupos son nilpotentes.

4. Si G es un grupo, entonces $K_{i}(G)$ es característico en $G$ para todo $i$.

En general, la relación de normalidad no es transitiva. La clausura transitiva de la normalidad es la subnormalidad.

Definición 1.15. Un subgrupo $H$ de un grupo $G$ se dice que es subnormal en $G$ cuando existe una serie $H=H_{0} \preccurlyeq H_{1} \preccurlyeq H_{2} \preccurlyeq \cdots \preccurlyeq H_{n}=G$ tal que $H_{i-1}$ es un subgrupo normal de $H_{i}$ para $1 \leqslant i \leqslant n$.

Recordamos también algunas caracterizaciones de grupos nilpotentes.

Teorema 1.16 ([DH92, Chapter A, Theorem 8.3]). Las siguientes afirmaciones sobre un grupo finito $G$ son equivalentes dos a dos:

1. Ges nilpotente.

2. Cada subgrupo maximal de G es normal.

3. Ges el producto directo de sus subgrupos de Sylow.

4. Todo factor principal de G es central.

5. Todos los subgrupos de G son subnormales. 


\subsection{Clases de grupos}

El lenguaje de clases de grupos permite presentar de una manera uniforme y generalizar resultados comunes a distintas propiedades invariantes por isomorfismos de grupos. Los libros de Doerk y Hawkes [DH92] y Ballester-Bolinches y Ezquerro [BBE06] son dos referencias básicas de la teoría de clases de grupos.

Definición 1.17. Una clase $\mathfrak{X}$ cuyos elementos son grupos se dice que es una clase de grupos si cada vez que $G$ es un grupo en $\mathfrak{X}$ y $H$ es un grupo isomorfo a $G$, se cumple que $H \in \mathfrak{X}$.

Observación 1.18. Cada propiedad de grupos que es cerrada bajo isomorfismos puede interpretarse como una clase de grupos formada por todos aquellos grupos que cumplen la propiedad.

Definición 1.19. Una clase de grupos $\mathfrak{X}$ se dice que es cerrada para extensiones cuando para cada grupo $G$ que posea un subgrupo normal $N$ tal que $N \in \mathfrak{X}$ y $G / N \in \mathfrak{X}$ se cumple que $G \in \mathfrak{X}$.

Definición 1.20. Decimos que una clase de grupos $\mathfrak{X}$ es cerrada para subgrupos (normales) cuando si $G$ es un grupo en $\mathfrak{X}$ y $H$ es un subgrupo (normal) de $G$, entonces $H$ pertenece a $\mathfrak{X}$.

Definición 1.21. Una clase de grupos $\mathfrak{X}$ se dice que es cerrada para cocientes cuando si $G \in \mathfrak{X}$ y $N$ es un subgrupo normal de $G$, entonces $G / N \in \mathfrak{X}$.

Ejemplos 1.22. La clase $\Im_{p}$ de todos los $p$-grupos para un primo $p$ y la clase $\mathfrak{S}$ de todos los grupos resolubles son ejemplos de clases cerradas para extensiones. El grupo simétrico $\Sigma_{3}$ de grado 3 muestra que la clase de todos los grupos nilpotentes no es cerrada para extensiones.

Son especialmente interesantes las siguientes clases de grupos.

Definición 1.23. Una clase de grupos $\mathfrak{\Im}$ se dice que es una formación si se cumplen las siguientes condiciones:

1. Si $N$ es subgrupo normal de $G$ y $G \in \mathfrak{F}$, entonces $G / N \in \mathfrak{F}$, es decir, $\mathfrak{F}$ es cerrada para cocientes.

2. Si $N_{1}, N_{2} \leqslant G$ y $G / N_{1}, G / N_{2} \in \mathfrak{F}$, entonces $G /\left(N_{1} \cap N_{2}\right) \in \mathfrak{F}$. 
Definición 1.24. Si $\mathfrak{F}$ es una formación no vacía y $G$ es un grupo, el subgrupo

$$
G^{\mathscr{F}}=\bigcap\{N \preccurlyeq G \mid G / N \in \mathfrak{F}\}
$$

se denomina $\mathfrak{Y}$-residual de $G$.

Proposición 1.25 ([DH92, Chapter II, Lemma 2.4]). Si F es una formación no vacía y $G$ es un grupo, entonces $G^{\widetilde{r}}$ es el menor subgrupo normal $K$ de $G$ tal que $G / K \in \mathfrak{F}$. Además, $G^{\widetilde{F}}$ es un subgrupo característico de $G$.

Ejemplos 1.26. Las clases $\mathfrak{A}$ de todos los grupos abelianos, $\mathfrak{\subseteq}$ de todos los grupos resolubles, $\mathfrak{U}$ de todos los grupos superresolubles y $\mathfrak{A}$ de todos los grupos nilpotentes son formaciones.

Definición 1.27. Una clase de grupos $\mathfrak{F}$ se dice que es una clase de Fitting cuando $\mathfrak{F}$ satisface las siguientes condiciones:

1. Si $N$ es un subgrupo normal de un grupo $G \in \mathfrak{F}$, entonces $N \in \mathfrak{F}$, es decir, $\mathfrak{F}$ es cerrada para subgrupos normales.

2. Si $G=N_{1} N_{2}$ es el producto de dos subgrupos normales $N_{1}$ y $N_{2}$ de $G$ y $N_{1}, N_{2} \in \mathfrak{F}$, entonces $G \in \mathfrak{F}$.

Definición 1.28. Si $\mathfrak{F}$ es una clase de Fitting no vacía y $G$ es un grupo, el $\mathfrak{\Im}$-radical $G_{\mathfrak{F}}$ de $G$ es el producto de todos los subgrupos normales de $G$ que pertenecen a $\mathfrak{F}$.

Proposición 1.29 ([DH92, Chapter II, Lemma 2.9]). El ₹r-radical $G_{\mathfrak{r}}$ es el mayor subgrupo normal de $G$ que pertenece a $\mathfrak{F}$. Además el $\mathfrak{F}$-radical $G_{\mathfrak{F}}$ de $G$ es un subgrupo característico de $G$.

Ejemplos 1.30. La clase $\subseteq$ de todos los grupos resolubles y la clase $\mathfrak{N}$ de todos los grupos nilpotentes son clases de Fitting. Lo mismo cabe decir con respecto a la clase $\mathfrak{C}$ de todos los grupos que son completamente reducibles (es decir, son triviales o producto directo de grupos simples) y tienen centro trivial (véase [Ros78, Exercise 419]). Sin embargo, la clase $\mathfrak{A}$ de todos los grupos abelianos y la clase $\mathfrak{U}$ de todos los grupos superresolubles son formaciones que no son clases de Fitting.

También hay ejemplos de clases de Fitting que no son formaciones. Por ejemplo, la clase $\mathfrak{D}$ formada por todos los grupos $G$ tales que para todo $g \in G$ se tiene que $\prod_{i=1}^{n} \operatorname{det}\left(g\right.$ sobre $\left.M_{i}\right)=1$, donde el producto 
se toma sobre los 3-factores principales $M_{1}, M_{2}, \ldots, M_{n}$ de una serie principal dada de $G$, es una clase de Fitting que no es una formación. Referimos al lector a [DH92, Chapter IX, Examples 2.14(b)] para más detalles.

En el caso de formaciones y clases de Fitting cerradas para extensiones, obtenemos los siguientes resultados:

Teorema 1.31. Si $\mathfrak{F}$ es una formación no vacía cerrada para extensiones y $G$ es un grupo, entonces $\left(G^{\mathscr{r}}\right)^{\mathscr{\mho}}=G^{\mathscr{\mho}}$.

Demostración. Notemos que $N=\left(G^{\widetilde{r}}\right)^{\widetilde{\Im}}$ es un subgrupo característico de $G^{\mathscr{F}}$, luego $N$ es un subgrupo característico en $G$. Por otra parte, $G / N$ tiene un subgrupo normal $G^{\widetilde{r}} / N$ de modo que $(G / N) /\left(G^{\widetilde{r}} / N\right) \cong$ $G / G^{\mathfrak{F}} \in \mathfrak{F}$ y $G^{\mathfrak{F}} / N \in \mathfrak{F}$. Por ser $\mathfrak{F}$ cerrada para extensiones, se sigue que $G / N \in \mathfrak{F}$. Pero entonces $G^{\mathfrak{F}} \leqslant N$. Se sigue que $N=G^{\mathfrak{r}}$.

Teorema 1.32. Si $\mathfrak{F}$ es una clase de Fitting no vacía cerrada para extensiones y $G$ es un grupo, entonces $\left(G / G_{\overparen{\mho}}\right)_{\mathfrak{F}}=1$.

Demostración. Denotemos por $N / G_{\mathfrak{\gamma}}=\left(G / G_{\mathfrak{\gamma}}\right)_{\mathfrak{F}}$. Notemos que $N / G_{\mathfrak{\mho}} \in$ $\mathfrak{F}$ y $G_{\mathfrak{\mho}} \in \mathfrak{F}$. Por ser $\mathfrak{F}$ cerrada para extensiones, se cumple que $N \in \mathfrak{F}$. Pero como $N$ es un subgrupo normal de $G$, se concluye que $N \leqslant G_{\mathfrak{F}}$. Así $N=G_{\mathfrak{r}}$.

\subsection{Grupos $p$-resolubles y $p$-nilpotentes}

En esta sección presentamos localizaciones de los conceptos de grupo resoluble y de grupo nilpotente. Dada una clase de grupos $\mathfrak{X}$, una localización de $\mathfrak{X}$ es una familia de clases de grupos $\left\{\mathfrak{X}_{p} \mid p \in \mathbb{P}\right\}$ de manera que $\mathfrak{X}=\bigcap_{p \in \mathbb{P}} \mathfrak{X}_{p}$. El siguiente concepto es una localización de la noción de grupo resoluble.

Definición 1.33 ([Hup67, Kapitel VI, Definition 1.3]). Sea $p$ un número primo. Un grupo finito $G$ se dice que es $p$-resoluble si cada factor principal de $G$ es o bien un $p$-grupo, o bien un $p^{\prime}$-grupo.

A partir de esta definición, el siguiente resultado es inmediato.

Teorema 1.34. Un grupo finito $G$ es resoluble si, y solo si, $G$ es p-resoluble para todo primo $p$. 
Definición 1.35. Sea $p$ un primo. La $p$-longitud de un grupo $p$-resoluble $G$ es el menor número de $p$-factores de una serie de $G$ cuyos factores sean $p$-grupos o $p^{\prime}$-grupos.

Así, un grupo tiene $p$-longitud a lo sumo 1 si $G / \mathrm{O}_{p^{\prime}}(G)$ tiene un $p$ subgrupo de Sylow normal, donde $\mathrm{O}_{p^{\prime}}(G)$ denota el mayor $p^{\prime}$-subgrupo normal de $G$.

A continuación presentamos el concepto de grupo $p$-nilpotente, que localiza la noción de grupo nilpotente. En [Hup67, Kapitel IV] y [Gor80, Chapter 7] se estudia este concepto en profundidad.

Definición 1.36 ([Hup67, Kapitel IV, Definition 4.1]). Sea $p$ un número primo. Un grupo finito $G$ se dice que es $p$-nilpotente si $G$ posee un $p^{\prime}$-subgrupo de Hall normal.

Teorema 1.37 (véase [Hup67, Kapitel IV, Satz 4.4]). Un grupo G es pnilpotente si, y solo si, todos los factores principales de $G$ de orden divisible por $p$ son centrales.

Como consecuencia del teorema 1.37 obtenemos el siguiente resultado.

Teorema 1.38. Un grupo $G$ es nilpotente si, y solo si, G es p-nilpotente para todo primo $p$.

El siguiente resultado se utilizará en el capítulo 5.

Teorema 1.39 (Burnside [Bur11, Section 243, Theorem II], [Gor80, Chapter 7, Theorem 4.3]). Sea p un primo. Si un p-subgrupo de Sylow de un grupo $G$ está centralizado por su normalizador, entonces $G$ es p-nilpotente.

Las técnicas para demostrar el teorema 1.39 permiten también demostrar el siguiente resultado, que se usará también en el capítulo 5.

Teorema 1.40 ([Gor80, Chapter 7, Theorem 4.4]). Si un p-subgrupo de Sylow $P$ de Ges abeliano y si $N=\mathrm{N}_{G}(P)$, entonces $P=\left(P \cap N^{\prime}\right) \times(P \cap Z(N))$. 



\section{Capítulo 2}

\section{Definiciones y propiedades básicas}

En este capítulo mostraremos los conceptos básicos sobre subgrupos solitarios y generalizaciones de este concepto.

\subsection{Subgrupos solitarios y subgrupos norma- les solitarios}

Definición 2.1. Un subgrupo $H \leqslant G$ se dice solitario cuando si $K \leqslant G$ y $H \cong K$, entonces $H=K$.

El concepto de subgrupo solitario fue introducido previamente en un artículo de Thévenaz [Thé93] bajo el nombre de subgrupo fuertemente característico. El nombre de subgrupo solitario que utilizamos en esta tesis fue introducido por Kaplan y Levy en [KL09, Definition 2]. Los subgrupos solitarios han sido estudiados por Kaplan y Levy en [KL09] (véase también [Lev14]), por Atanasov y Foguel en [AF12] y por Tărnăuceanu en [Tăr12b] (véase también [Tăr12a]).

Una propiedad de inmersión de subgrupos ofuntor de inmersión de subgrupos $f$ asigna a cada grupo $G$ una familia de subgrupos $f(G)$ de manera que si $\alpha$ es un isomorfismo de grupos, $f\left(G^{\alpha}\right)=\left\{S^{\alpha} \mid S \in f(G)\right\}$. Si $S \in f(G)$, diremos que $S$ es un $f$-subgrupo de $G$ o que $S$ satisface la propiedad de inmersión $f$ en $G$. Ejemplos clásicos de propiedades de inmersión incluyen la propiedad de ser subgrupo o ser subgrupo normal. 
Dada una propiedad de inmersión de subgrupos $f$, podemos considerar los $f$-subgrupos que no son isomorfos a ningún otro $f$-subgrupo.

Definición 2.2. Dada una propiedad de inmersión de subgrupos $f$, un $f$-subgrupo $H$ de un grupo $G$ se dice $f$-solitario en $G$ si dado un $f$-subgrupo $K$ de $G$ de manera que $H \cong K$, se tiene que $H=K$.

Cuando $f=s$ es simplemente la propiedad de ser subgrupo, los subgrupos $s$-solitarios son simplemente los subgrupos solitarios. Para el caso en que la propiedad de inmersión $f$ sea la normalidad, obtenemos la noción de subgrupo normal solitario introducida por Kaplan y Levy en [KL09].

Definición 2.3 ([KL09, Definition 2]). Un subgrupo $H \preccurlyeq G$ se llama normal solitario en $G$ si ningún otro subgrupo de $G$ isomorfo a $H$ es normal en $G$. En otras palabras, un subgrupo $H \Vdash G$ es normal solitario cuando si $H \leqslant G$ y $H \cong K$, entonces $H \nsubseteq G$, es decir, si $K \unlhd G$ y $H \cong K$, entonces $H=K$.

Más adelante (véase la definición 3.6 en la página 34) presentaremos el concepto de subgrupo subnormal solitario, que corresponde al caso en que la propiedad $f$ sea la subnormalidad.

Ejemplo 2.4. Sea $G=\Sigma_{4}$ el grupo simétrico de grado 4. Entonces $\mathrm{F}(G)=V_{4}$ y $S=\langle(1,2)(3,4),(3,4)\rangle \cong V_{4}$, con lo que $V_{4}$ no es un subgrupo solitario de $G$. Sin embargo, $V_{4}$ es un subgrupo normal solitario de $G$, ya que es el único subgrupo normal de $G$ de orden 4 .

La siguiente propiedad justifica el nombre de subgrupo fuertemente característico dado por Thévenaz en [Thé93].

Proposición 2.5. Todo subgrupo solitario (normal solitario) de G es característico en $\mathrm{G}$.

Demostración. Sean $H$ un subgrupo solitario (normal solitario) de $G$ y $\alpha \in \operatorname{Aut}(G)$. Entonces $H^{\alpha} \cong H$. Por ser $H$ solitario (normal solitario) en $G, H^{\alpha}=H$. Por tanto, $H$ es característico en $G$.

Los subgrupos de Hall normales son también solitarios, como Atanasov y Foguel ponen de manifiesto en [AF12]. De hecho, es un resultado muy conocido que si un grupo posee un $\pi$-subgrupo de Hall normal, entonces este es el único $\pi$-subgrupo de Hall del grupo. Presentamos aquí una prueba de este hecho por completitud. 
Proposición 2.6 ([AF12, Proposition 2.1]). Si H es un subgrupo normal de $G$ de manera que $\operatorname{mcd}(|H|,|G / H|)=1$, entonces $H$ es solitario en $G$.

Demostración. Sea $K$ un subgrupo de $G$ isomorfo a $H$. Entonces $H K$ es un subgrupo de $G$. Puesto que $K$ y $H$ tienen el mismo orden, todos los factores primos de $|H K|$ son factores primos de $|H|$. Como $\operatorname{mcd}(|H|,|G / H|)=1$, se deduce que $\operatorname{mcd}(|H K|,|G / H|)=1$. Supongamos que $H \neq H K$. Entonces $|H|$ es divisor de $|G|,|H K|$ es divisor de $|G|$ y $|H|<|H K|$. Existe un número primo $p$ divisor de $|H K| /|H|$, lo que implica que $p$ es divisor de $|H|$ y $|G / H|$. Esto contradice la suposición $\operatorname{mcd}(|H|,|G / H|)=1$. Así, $H K=H$, lo que implica que $H=K$. Por lo tanto, $H$ es solitario en $G$.

Como consecuencia inmediata del teorema anterior, tenemos los siguientes dos corolarios.

Corolario 2.7 ([AF12, Corollary 2.2]). Si $G=H_{1} \times H_{2} y \operatorname{mcd}\left(\left|H_{1}\right|,\left|H_{2}\right|\right)=$ 1 , entonces $H_{1}$ y $\mathrm{H}_{2}$ son solitarios en $G$.

Corolario 2.8 ([AF12, Corollary 2.3]). Si G $=H_{1} \times H_{2} \times \cdots \times H_{n}$ y para $i, j \in\{1,2, \ldots, n\}, i \neq j$, se tiene que $\operatorname{mcd}\left(\left|H_{i}\right|,\left|H_{j}\right|\right)=1$, entonces $H_{i}$ es solitario en $G$ para todo $i \in\{1,2, \ldots, n\}$.

Ejemplo 2.9. Si $G=\Sigma_{3} \times C_{3}$, donde $\Sigma_{3}$ denota el grupo simétrico de grado 3 y $C_{3}$ el grupo cíclico de orden 3 , se tiene que el subgrupo alternado $A_{3}$ es un subgrupo solitario de $\Sigma_{3}$ y $\Sigma_{3}$ es un subgrupo solitario de $G$. Sin embargo, $A_{3}$ no es un subgrupo solitario de $G$, ya que $A_{3}$ es isomorfo a $C_{3}$. Esto prueba que la relación de ser un subgrupo solitario no es una relación transitiva. Este mismo ejemplo muestra que la relación de ser subgrupo normal solitario tampoco es transitiva.

\subsection{Subgrupos solitarios para cocientes}

Parece natural considerar el concepto dual al de subgrupo solitario para cocientes. Tărnăuceanu introdujo en [Tăr12b] la noción de subgrupo solitario para cocientes. Este mismo concepto ya fue introducido en nuestro trabajo de investigación de tercer ciclo [LC11] bajo el nombre de subgrupo dual-solitario. 
Definición 2.10 ([Tăr12b]). Un subgrupo normal $N$ de un grupo $G$ se dice que es solitario para cocientes en $G$ si cada vez que $K$ es un subgrupo normal de $G$ y $G / N$ es isomorfo a $G / K$, se tiene que $N=K$.

Proposición 2.11. Un subgrupo solitario para cocientes de G es característico en $G$.

Demostración. Supongamos que $N$ es un subgrupo solitario para cocientes de $G$ y que $\alpha$ es un automorfismo de $G$. Entonces $N^{\alpha}$ es un subgrupo normal de $G$. Consideremos el homomorfismo $\beta: G \longrightarrow G / N^{\alpha}$ definido mediante $g^{\beta}=g^{\alpha} N^{\alpha}$. Este homomorfismo tiene como núcleo $N$ y es claramente un epimorfismo, con lo que $G / N \cong G / N^{\alpha}$. Por ser $N$ solitario para cocientes, se tiene que $N=N^{\alpha}$, como se deseaba.

La noción de subgrupo solitario para cocientes es esencialmente distinta de la noción de subgrupo solitario o la de subgrupo normal solitario.

Ejemplo 2.12. Sea $G=C_{4} \times C_{2}=\left\langle a, b \mid a^{4}=b^{2}=[a, b]=1\right\rangle$ el producto directo de un grupo cíclico de orden 4 y un grupo cíclico de orden 2 . El subgrupo $H=\left\langle a^{2}, b\right\rangle$ es solitario, ya que es el único subgrupo abeliano elemental de orden 4 , pero no es solitario para cocientes, ya que $G / H \cong$ $C_{2}$ y $G /\langle a\rangle \cong C_{2}$. Por otra parte, $K=\left\langle a^{2}\right\rangle=\Phi(G)$ es un subgrupo que es solitario para cocientes, ya que es el único subgrupo normal que da cociente $C_{2} \times C_{2}$, pero no es solitario, puesto que $K \cong\langle b\rangle$.

Igual que vimos en la proposición 2.6, los subgrupos de Hall normales son también solitarios para cocientes. La demostración es análoga a la de la proposición 2.6 y por eso la omitimos.

Proposición 2.13. Si H es un subgrupo normal de $G y \operatorname{mcd}(|H|,|G / H|)=1$, entonces $H$ es solitario para cocientes en $G$.

Los subgrupos solitarios para cocientes se comportan bien con respecto a cocientes.

Proposición 2.14. Supongamos que $N$ es un subgrupo normal de un grupo $G$ y que $S$ es un subgrupo solitario para cocientes de $G$ de manera que $N \leqslant S$. Entonces $S / N$ es un subgrupo solitario para cocientes de G/N.

Demostración. Supongamos que $T / N$ es un subgrupo normal de $G / N$ de manera que $(G / N) /(S / N) \cong(G / N) /(T / N)$. Entonces $G / S \cong G / T$ y, por ser $S$ solitario para cocientes, $S=T$. 
Observación 2.15. Como se indica en [Tăr12a] y contrariamente a lo que se afirma en [Tăr12b, Proposition 2.3], no podemos afirmar que la imagen por un epimorfismo de un subgrupo solitario para cocientes sea un subgrupo solitario para cocientes. Sea $\Sigma_{3}=\langle a, b| a^{2}=b^{3}=$ $\left.1, b^{a}=b^{-1}\right\rangle$ un grupo simétrico de grado 3 y sea $D_{10}=\langle c, d| c^{2}=$ $\left.d^{5}=1, d^{c}=d^{-1}\right\rangle$ un grupo diédrico de orden 10 . Tomemos $G=\Sigma_{3} \times$ $D_{10}$ el producto directo de estos grupos. Consideremos $N=\langle b, d\rangle$. El subgrupo $S=\langle c, d\rangle$ es solitario para cocientes en $G$, pero su imagen en $G / N, H_{1} / N=\langle b, c, d\rangle / N$, no es un subgrupo solitario para cocientes, ya que $H_{2} / N=\langle a b, c, d\rangle / N$ es un subgrupo normal de $G / N$ de manera que $(G / N) /\left(H_{1} / N\right) \cong(G / N) /\left(H_{2} / N\right) \cong C_{2}$, pero $H_{1} / N \neq H_{2} / N$.

Por último, mostramos que la relación de ser subgrupo solitario para cocientes es transitiva.

Proposición 2.16 ([Tăr12b, Proposition 2.2]). Sea G un grupo y $H, K$ dos subgrupos normales de $G$ con $K \leqslant H$. Si $H$ es solitario para cocientes en $G y$ $K$ es solitario para cocientes en $H$, entonces $K$ es solitario para cocientes en $G$.

Demostración. Sea $K_{1}$ un subgrupo normal de $G$ tal que $G / K_{1} \cong G / K$ y consideremos un isomorfismo $f: G / K \longrightarrow G / K_{1}$. Denotemos $H_{1} / K_{1}=$ $f(H / K)$, donde $H_{1}$ es un subgrupo normal de $G$ de modo que $K_{1} \leqslant H_{1}$. Se sigue que

$$
G / H \cong(G / K) /(H / K) \cong\left(G / K_{1}\right) /\left(H_{1} / K_{1}\right) \cong G / H_{1} .
$$

Esto nos lleva a que $H_{1}=H$. Se obtiene que $H / K_{1} \cong H / K$ y, por tanto, $K_{1}=K$. Se concluye que $K$ es un subgrupo solitario para cocientes de $G$. 



\section{Capítulo 3}

\section{Propiedades reticulares}

En este capítulo vemos que los subgrupos solitarios y los subgrupos solitarios para cocientes forman retículos con la inclusión de subgrupos, como muestran Kaplan y Levy en [KL09] y Tărnăuceanu en [Tăr12b], respectivamente (el último resultado también forma parte de nuestro trabajo de investigación de tercer ciclo [LC11]). Nuestra principal aportación a este estudio consiste en mostrar que estos retículos no son en general subretículos del retículo de subgrupos normales. También vemos que el conjunto parcialmente ordenado de los subgrupos normales solitarios con la inclusión de subgrupos no constituye un retículo. Esto motiva la introducción del concepto de subgrupo subnormal solitario. Los subgrupos subnormales solitarios conformarán un retículo con la inclusión de subgrupos, que tampoco será un subretículo del retículo de subgrupos normales. Estos resultados aparecen en [ERL].

\subsection{Observaciones sobre el retículo de sub- grupos solitarios}

Comenzaremos por mostrar que el conjunto de subgrupos solitarios de un grupo constituye un retículo con la inclusión de subgrupos.

Teorema 3.1 ([KL09, Theorem 25]). Si G es un grupo, entonces el conjunto $\operatorname{Sol}(G)$ formado por todos los subgrupos solitarios de $G$ es un retículo con respecto a la inclusión de subgrupos. 
Demostración. Sean $S_{1}$ y $S_{2}$ solitarios en $G$. Entonces, en particular, $S_{1}$ y $S_{2}$ son normales y así $S_{1} S_{2}$ es un subgrupo de $G$. Supongamos que $T \leqslant G, T \cong S_{1} S_{2}$. Entonces $T$ contiene subgrupos $T_{1}$ y $T_{2}$, que son isomorfos a $S_{1}$ y $S_{2}$ respectivamente, y $T=T_{1} T_{2}$. El carácter solitario de $S_{1}$ y $S_{2}$ en $G$ implica inmediatamente que $T=S_{1} S_{2}$. Por lo tanto, $S_{1} S_{2}$ es solitario en $G$. Además, cualquier subgrupo que contiene tanto a $S_{1}$ como a $S_{2}$ debe contener a $S_{1} S_{2}$. Por consiguiente, $S_{1} S_{2}$ es el supremo de $\left\{S_{1}, S_{2}\right\}$. Demostramos ahora que el ínfimo de $\left\{S_{1}, S_{2}\right\}$ también existe. Primero notemos que 1 es un subgrupo solitario de $G$ incluido tanto en $S_{1}$ como en $S_{2}$, con lo que el conjunto de subgrupos solitarios de $G$ contenidos tanto en $S_{1}$ como en $S_{2}$ no es vacío. Tomemos ahora el subgrupo generado por todos los subgrupos solitarios de $G$ que están contenidos tanto en $S_{1}$ como en $S_{2}$. Entonces, por lo que hemos demostrado arriba, este subgrupo es solitario en $G$ y está contenido en $S_{1}$ y $S_{2}$. Este subgrupo es el ínfimo de $\left\{S_{1}, S_{2}\right\}$. Notemos que 1 y $G$ son, respectivamente, el mínimo y el máximo de $\operatorname{Sol}(G)$, respectivamente.

Sin embargo, este retículo no es, en general, un subretículo del retículo de subgrupos normales, donde el supremo de dos subgrupos normales coincide con el subgrupo generado por ellos y el ínfimo, con su intersección.

Ejemplo 3.2 ([ERL, Example 2.1]). Sea $G$ un producto directo de un grupo simétrico $\Sigma_{3}=\left\langle a, b \mid a^{2}=b^{3}=1, b^{a}=b^{-1}\right\rangle$ de grado 3 y un grupo cíclico $C_{3}=\left\langle c \mid c^{3}=1\right\rangle$ de orden 3 . Este grupo tiene dos subgrupos solitarios, $\Sigma_{3}=\langle a, b\rangle$ y el 3-subgrupo de Sylow normal $\langle b, c\rangle \cong C_{3} \times C_{3}$, cuya intersección es $\langle b\rangle$, un grupo cíclico de orden 3 que es claramente no solitario en $G$. Por tanto, la intersección de dos subgrupos solitarios no es necesariamente un subgrupo solitario.

El siguiente teorema puede verse como el dual del teorema 3.1 para subgrupos solitarios para cocientes.

Teorema 3.3 ([Tăr12b, Proposition 2.1], [LC11, Teorema 3.4]). Si G es un grupo, el conjunto QSol $(G)$ formado por todos los subgrupos solitarios para cocientes de $G$ con la inclusión es un retículo.

Demostración. Supongamos que $A$ y $B$ son dos subgrupos solitarios para cocientes. Veamos en primer lugar que $A \cap B$ es un subgrupo solitario 


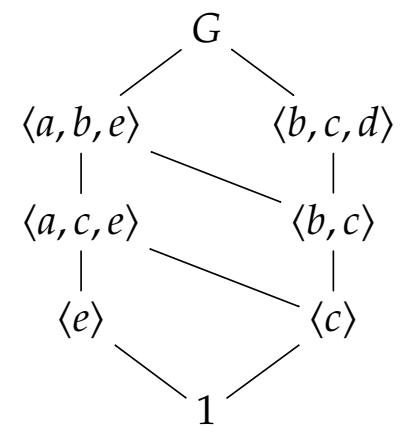

Figura 3.1: Retículo de subgrupos solitarios para cocientes del grupo del ejemplo 3.4

para cocientes. Supongamos que $K$ es un subgrupo normal de $G$ y que $G /(A \cap B)$ es isomorfo a $G / K$. Entonces $G / K$ posee subgrupos normales $A_{1} / K$ y $B_{1} / K$ de manera que $A_{1} \cap B_{1}=K$ y $G / A_{1} \cong(G / K) /\left(A_{1} / K\right) \cong G / A$ y $G / B_{1} \cong(G / K) /\left(B_{1} / K\right) \cong G / B$. Por ser $A$ y $B$ solitarios para cocientes, se sigue que $A_{1}=A$ y $B_{1}=B$. Pero entonces $K=A_{1} \cap B_{1}=A \cap B$.

Consideremos ahora el conjunto de todos los subgrupos solitarios para cocientes de $G$ que contienen $A$ y $B$. Este conjunto no es vacío porque $G$ es uno de estos subgrupos. Además, por lo visto en el párrafo anterior, la intersección de todos ellos es un subgrupo solitario para cocientes de $G$ que contiene $A$ y $B$. Este subgrupo resulta ser el supremo de $\{A, B\}$. Concluimos que los subgrupos solitarios para cocientes de $G$ constituyen un retículo.

Ejemplo 3.4 ([ERL, Example 2.2]). Al igual que ocurría con el retículo de los subgrupos solitarios, el retículo de los subgrupos solitarios para cocientes no es, en general, un subretículo del retículo de los subgrupos normales. Para ello consideremos un grupo extraespecial $E=\langle a, b\rangle$ de orden 27 y exponente 3 . Denotemos por $c=[a, b]$. Este grupo $E$ posee un automorfismo $d$ de orden 2 dado por $a^{d}=a$ y $b^{d}=b^{2}$, entonces se tiene que $c^{d}=c^{2}$. Sea $H=[E]\langle d\rangle$ el correspondiente producto semidirecto. Consideremos ahora un grupo cíclico $C=\langle e\rangle$ de orden 3 y el producto directo $G=H \times C$. Se puede comprobar (por ejemplo, con la ayuda de GAP [GAP15]) que los subgrupos solitarios para cocientes de $G$ son $1,\langle c\rangle,\langle e\rangle,\langle a, c, e\rangle,\langle b, c\rangle,\langle a, b, e\rangle,\langle b, c, d\rangle$ y $G$. La figura 3.1 muestra el 


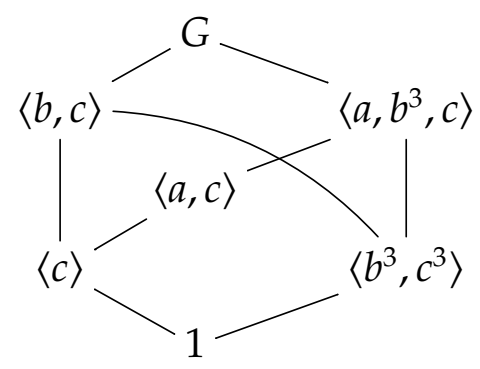

Figura 3.2: Conjunto parcialmente ordenado de los subgrupos normales solitarios del grupo del ejemplo 3.5

diagrama de Hasse de este retículo. El producto de $\langle c\rangle$ y $\langle e\rangle$ no es un subgrupo solitario para cocientes de $G$.

La situación es aún peor con subgrupos normales solitarios, ya que no forman un retículo en general.

Ejemplo 3.5. Sea

$$
G=\left\langle a, b, c \mid a^{2}=b^{9}=c^{9}, b^{a}=b, c^{a}=c^{8}, c^{b}=c^{7}\right\rangle .
$$

Los subgrupos normales solitarios de $G$ son $1,\left\langle b^{3}, c^{3}\right\rangle,\langle c\rangle,\langle a, c\rangle,\left\langle a, b^{3}, c\right\rangle$, $\langle b, c\rangle$ y $G$, obtenidos con ayuda del sistema de álgebra computacional GAP [GAP15]. El conjunto parcialmente ordenado de estos subgrupos normales solitarios está representado en la figura 3.2. Vemos que el subconjunto $\left\{\left\langle b^{3}, c^{3}\right\rangle,\langle c\rangle\right\}$ no posee supremo en el conjunto parcialmente ordenado de los subgrupos normal solitarios de $G$, mientras que $\left\{\left\langle a, b^{3}, c\right\rangle,\langle b, c\rangle\right\}$ no tiene ínfimo.

Parece claro que uno de los principales obstáculos para que el conjunto parcialmente ordenado de los subgrupos normales solitarios sea un retículo es el hecho de que la normalidad no es una relación transitiva en general. La clausura transitiva de la normalidad es la subnormalidad (véase la definición 1.15 en la página 19).

Definición 3.6 ([ERL, Definition 2.4]). Un subgrupo $H$ de un grupo $G$ se dice que es un subgrupo subnormal solitario de $G$ cuando $H$ es un subgrupo subnormal de $G$ y si $K$ es otro subgrupo subnormal de $G$ isomorfo a $H$, entonces $K=H$. 
Esta definición se corresponde con la noción de subgrupo $f$-solitario (véase la definición 2.2 en la página 26) cuando la propiedad de inmersión $f$ es la subnormalidad.

Obviamente, los subgrupos subnormales solitarios son característicos (vale el mismo argumento de la demostración de la proposición 2.5 en la página 26). El siguiente resultado es una consecuencia inmediata de la definición.

Proposición 3.7 ([ERL, Proposition 3.5]). Sea H un subgrupo de un grupo G.

1. Si $H$ es solitario en $G$, entonces $H$ es subnormal solitario en $G$.

2. Si $H$ es subnormal solitario en $G$, entonces $H$ es normal solitario en $G$.

Claramente, en grupos nilpotentes, las nociones de subgrupo solitario y subgrupo subnormal solitario coinciden, ya que todo subgrupo es subnormal, mientras que en los grupos donde la normalidad es una relación transitiva, los llamados T-grupos (por ejemplo, en los grupos abelianos), los subgrupos subnormales solitarios y los subgrupos normales solitarios coinciden. En [BBERA10, Chapter 2] se puede hallar más información sobre los T-grupos.

Los recíprocos de las implicaciones de la proposición 3.7 son falsos:

Ejemplo 3.8 ([ERL, Example 2.6]). El grupo diédrico $D_{8}=\langle a, b| a^{4}=$ $\left.b^{2}=(a b)^{2}=1\right\rangle$ de orden 8 tiene un subgrupo normal solitario $\left\langle a^{2}\right\rangle=$ $\Phi\left(D_{8}\right)$ que no es subnormal solitario en $D_{8}$, ya que este grupo cuenta con cinco subgrupos subnormales cíclicos de orden 2.

Ejemplo 3.9 ([ERL, Example 2.7]). El grupo simétrico $\Sigma_{4}$ de orden 4 tiene un subgrupo subnormal solitario $V_{4}=\langle(1,2)(3,4),(1,3)(2,4)\rangle$ que no es solitario en $\Sigma_{4}$, porque $V_{4}$ es isomorfo al subgrupo no subnormal $\langle(1,2),(3,4)\rangle$.

Sin embargo, se tiene el siguiente resultado.

Teorema 3.10 ([ERL, Theorem 2.8]). El conjunto parcialmente ordenado de todos los subgrupos subnormales solitarios de un grupo con la inclusión es un retículo. 


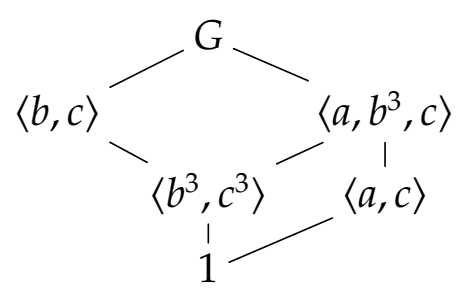

Figura 3.3: Retículo de subgrupos subnormales solitarios del grupo de los ejemplos 3.5 y 3.11

Demostración. Supongamos que $S_{1}$ y $S_{2}$ son subgrupos subnormales solitarios en $G$. Entonces $S_{1}$ y $S_{2}$ son subgrupos normales de $G$. Supongamos que $T$ es un subgrupo subnormal de $G$ isomorfo a $S=S_{1} S_{2}$. Notemos que $S_{1}$ y $S_{2}$ son subgrupos normales de $S$. Por lo tanto, $T$ contiene subgrupos normales $T_{1}$ y $T_{2}$ tales que $S_{1} \cong T_{1}$ y $S_{2} \cong T_{2}$. Como $T_{1}$ y $T_{2}$ son normales en $G$ y $T$ es subnormal en $G$, tenemos que $T_{1}$ y $T_{2}$ son subgrupos subnormales de $G$. Como $S_{1}$ y $S_{2}$ son subnormales solitarios, obtenemos que $S_{1}=T_{1}$ y $S_{2}=T_{2}$. En particular, $S=T$. Esto implica que $S$ es subnormal solitario y, obviamente, $S$ es el supremo de $\left\{S_{1}, S_{2}\right\}$ en el conjunto parcialmente ordenado de todos los subgrupos subnormales solitarios de $G$.

El argumento para demostrar que un conjunto de dos subgrupos subnormales solitarios posee un ínfimo es el mismo que en el teorema 3.1 .

Ejemplo 3.11 ([ERL, Example 2.9]). En el grupo del ejemplo 3.5, cuyo conjunto parcialmente ordenado de subgrupos normales solitarios aparece representado en la figura 3.2, el único subgrupo normal solitario que no es subnormal solitario es $\langle c\rangle$. El diagrama de Hasse del retículo de subgrupos subnormales solitarios de este grupo aparece representado en la figura 3.3. La intersección $\langle b, c\rangle \cap\left\langle a, b^{3}, c\right\rangle=\left\langle b^{3}, c\right\rangle$ no es un subgrupo subnormal solitario de $G$. Este ejemplo también muestra que el retículo de todos los subgrupos subnormales solitarios no es, en general, un subretículo del retículo de subgrupos normales. 


\section{Capítulo 4}

\section{Subgrupos solitarios y clases de grupos}

En este capítulo estudiamos condiciones en que los radicales asociados a clases de Fitting no vacías y los residuales asociados a formaciones no vacías son subgrupos (subnormales) solitarios o subgrupos solitarios para cocientes. Esto nos permite generalizar y mejorar algunos resultados de Kaplan y Levy [KL09] y de Tărnăuceanu [Tăr12b]. Por otra parte, una pregunta natural en este contexto es si los subgrupos solitarios o normales solitarios pueden verse como radicales para clases de Fitting adecuadas y si los subgrupos solitarios para cocientes pueden verse como residuales para formaciones adecuadas. Respondemos negativamente a estas cuestiones. Nuestras aportaciones en esta línea aparecen en [ERL].

\subsection{Subgrupos solitarios de tipo radical y re- sidual}

Comenzamos por asociar a cada grupo $G$ y a cada clase de grupos $\mathfrak{X}$ un subgrupo solitario $B_{\mathfrak{X}}(G)$.

Teorema 4.1 ([KL09, Lemma 3]). Dada una clase de grupos no vacía $\mathfrak{X}$, el subgrupo $B_{\mathfrak{X}}(G)$ generado por todos los subgrupos de $G$ en $\mathfrak{X}$ es solitario en $G$.

Demostración. Consideremos la familia $\mathcal{S}=\left\{S_{1}, \ldots, S_{k}\right\}$ formada por todos los $\mathfrak{X}$-subgrupos de $G$. Supongamos que $H \cong B_{\mathfrak{X}}(G)$, entonces 
$H$ contiene exactamente $k \mathfrak{X}$-subgrupos. Por tanto, $H$ contiene todos los $\mathfrak{X}$-subgrupos de $G$. Se sigue que $B_{\mathfrak{X}}(G) \leqslant H$, de donde se sigue la igualdad por consideraciones sobre órdenes.

Si tenemos en cuenta los subgrupos solitarios subnormales introducidos en la definición 3.6, obtenemos:

Teorema 4.2 ([ERL, Theorem 3.1]). Sea $\mathfrak{X}$ una clase de grupos no vacía. El subgrupo $S_{\mathfrak{X}}(G)$ generado por todos los subgrupos subnormales de $G$ en $\mathfrak{X}$ es un subgrupo subnormal solitario de $G$.

Demostración. Sea $\mathcal{S}=\left\{S_{1}, \ldots, S_{k}\right\}$ el conjunto de todos los subgrupos subnormales de $G$ en $\mathfrak{X}$. Como $\mathcal{S}$ es invariante por conjugación, tenemos que $S_{\mathfrak{x}}(G)$ es un subgrupo normal de $G$. Sea $H$ un subgrupo subnormal de $G$ isomorfo a $S_{\mathfrak{x}}(G)$. Entonces $H$ contiene exactamente $k$ subgrupos subnormales de $G$ en $\mathfrak{X}$, es decir, todos los subgrupos en $\mathcal{S}$ están contenidos en $H$. De ello se desprende que $H=S_{\mathfrak{x}}(G)$.

Si $\mathfrak{X}$ es una clase de Fitting no vacía, se obtiene que el $\mathfrak{X}$-radical de un grupo $G$, es decir, el subgrupo generado por todos los subgrupos subnormales de $G$ en $\mathfrak{X}$ (véase la definición 1.28 en la página 21), es un subgrupo subnormal solitario de $G$, en particular, es un subgrupo normal solitario de G. Esto confirma el resultado de [KL09, Lemma 15].

Teorema 4.3 ([ERL, Theorem 3.2], [KL09, Lemma 15]). Sea $\mathfrak{F}$ una clase de Fitting no vacía y sea $G$ un grupo. Entonces el $\mathfrak{F}$-radical $G_{\mathfrak{\gamma}}$ de $G$ es un subgrupo subnormal solitario de $G$.

Los subgrupos solitarios para cocientes cumplen la siguiente propiedad dual de la del teorema 4.1:

Teorema 4.4 ([ERL, Theorem 3.3]). Sea $\mathfrak{X}$ una clase de grupos. Entonces la intersección de todos los subgrupos normales $N$ de $G$ de manera que $G / N \in \mathfrak{X}$ es un subgrupo solitario para cocientes de $G$.

Demostración. Sea $\mathcal{N}=\left\{N_{1}, \ldots, N_{k}\right\}$ el conjunto de todos los subgrupos normales de $G$ con cociente en $\mathfrak{X}$. Sea $H$ la intersección de todos estos subgrupos y supongamos que $G / K$ es isomorfo a $G / H$. Entonces $G / K$ posee subgrupos normales $K_{1} / K, \ldots, K_{k} / K$ tal que $K_{1} \cap \cdots \cap K_{k}=K$ y $G / K_{i} \in \mathfrak{X}$ para $1 \leqslant i \leqslant k$. Se sigue que estos subgrupos deben ser exactamente los elementos de $\mathcal{N}$. Por lo tanto, $K=H$. 
Dado que, para una formación no vacía $\mathfrak{\&}, G^{\mathfrak{F}}$ es la intersección de todos los subgrupos normales de $G$ con cociente en $\mathfrak{F}$ (véase la definición 1.24 en la página 21), tenemos:

Corolario 4.5 ([ERL, Corollary 3.4]). Sea F una formación no vacía y sea $G$ un grupo. Entonces el $\mathfrak{F}$-residual $G^{\mathfrak{F}}$ de $G$ es un subgrupo solitario para cocientes de $G$.

Este resultado se puede utilizar para dar una descripción de grupos libres de subgrupos solitarios para cocientes, es decir, grupos $G$ para los cuales los únicos subgrupos solitarios para cocientes son $G$ y 1 . Esto mejora el resultado de [Tăr12b, Theorem 3.7], donde se demuestra que estos grupos son perfectos o abelianos elementales.

Teorema 4.6 ([ERL, Theorem 3.5]). Las siguientes afirmaciones son equivalentes para un grupo $G$ :

1. Ges característicamente simple.

2. G es libre de subgrupos solitarios para cocientes.

3. Ges un producto directo de copias de un grupo simple $S$.

Demostración. La equivalencia entre los enunciados 1 y 3 es muy conocida. Supongamos que $G$ es libre de subgrupos solitarios para cocientes. Sea $M$ un subgrupo normal maximal de $G$, entonces $S=G / M$ es un grupo simple y consideremos la clase $\mathfrak{F}=\mathrm{D}_{0}(1, S)$ de todos los grupos que pueden ser expresados como un producto directo de copias de $S$ junto con el grupo trivial. Si $S$ es un grupo simple no abeliano, esta clase es una formación por [DH92, Chapter II, Example 2.13], y si $S \cong C_{p}, p$ primo, esta es la clase de todos $p$-grupos abelianos elementales, que es también una formación. Como $G^{\widetilde{r}} \leqslant M<G$, se tiene que $G^{\mathscr{\mathscr { F }}}=1$, en otras palabras, $G \in \mathfrak{F}$ y $G$ es un producto directo de copias de un grupo simple $S$. Por último, como los subgrupos solitarios para cocientes son subgrupos característicos, si G es característicamente simple, entonces $G$ no puede poseer subgrupos solitarios para cocientes propios. 


\subsection{Radicales solitarios para cocientes y resi- duales solitarios}

En la sección anterior hemos visto que los radicales para una clase de Fitting no vacía son subnormales solitarios y los residuales para una formación no vacía son solitarios para cocientes. En esta sección estudiamos condiciones para que un residual sea solitario y un radical sea solitario para cocientes. Kaplan y Levy han probado el siguiente resultado para subgrupos solitarios y subgrupos normales solitarios. Extendemos el resultado a subgrupos subnormales solitarios.

Teorema 4.7 (véase [KL09, Lemma 22]). Sea F una formación de grupos no vacía cerrada para extensiones y subgrupos (normales), entonces el $\mathfrak{F}$-residual $G^{\mathfrak{r}}$ es solitario (subnormal solitario) en $G$.

Demostración. Veremos la demostración para formaciones cerradas para subgrupos normales. Notemos que las formaciones cerradas para subgrupos normales son cerradas también para subgrupos subnormales. El resultado para formaciones cerradas para subgrupos se prueba análogamente sustituyendo subgrupo normal o subgrupo subnormal por subgrupo.

Supongamos que $K$ es un subgrupo subnormal de $G$ de manera que $K \cong G^{\mathscr{r}}$. Como $\left(G^{\mathfrak{F}}\right)^{\mathfrak{F}}=G^{\mathfrak{F}}$ por ser $\mathfrak{F}$ cerrada para extensiones en virtud del teorema 1.31 (página 22), se sigue que $K^{\mathscr{\&}}=K$ ya que $K \cong G^{\mathscr{\&}}$. Supongamos que $K \neq G^{\mathscr{F}}$. Entonces $K /\left(G^{\mathscr{夭}} \cap K\right) \cong G^{\mathscr{\mho}} K / G^{\mathscr{F}}$, que es un subgrupo subnormal de $G / G^{\mathscr{F}}$. Como $G / G^{\mathscr{F}} \in \mathfrak{F}$ y $\mathfrak{F}$ es cerrada para subgrupos normales, en particular, para subgrupos subnormales, se tiene que $G^{\widetilde{F}} K / G^{\mathscr{F}} \in \mathfrak{F}$. Entonces $K=K^{\mathscr{F}} \leqslant G^{\mathscr{F}} \cap K$, es decir, $K \leqslant G^{\mathscr{F}}$. Por tanto, $K=G^{\widetilde{r}}$. Esto prueba que $G^{\widetilde{\mathscr{}}}$ es un subgrupo subnormal solitario de $G$.

Podríamos preguntarnos si se puede prescindir de la condición de clausura para extensiones y subgrupos (normales). Más precisamente, qué se puede decir de una formación no vacía en la que, dado un grupo $G$, el $\mathfrak{F}$-residual de $G$ es siempre un subgrupo solitario (respectivamente, subnormal solitario) de $G$. Hemos obtenido el siguiente resultado para formaciones $\mathfrak{F}$ no vacías que cumplen que $(G \times H)^{\mathscr{\mho}}=G^{\mathscr{}} \times H^{\widetilde{\mho}}$ por cada dos grupos $G$ y $H$. Todas las formaciones no vacías contenidas en los grupos resolubles satisfacen esta condición, como se muestra 
en Doerk y Hawkes [DH78] (véase también [DH92, Chapter IV, Theorem 1.18]).

Teorema 4.8 ([ERL, Theorem 3.7]). Supongamos que F es una formación no vacía que satisface $(G \times H)^{\mathscr{\mho}}=G^{\widetilde{r}} \times H^{\mathscr{\mho}}$ para cada dos grupos $G$ y $H$. Supongamos, además que, dado un grupo $G$, el $\mathfrak{F}$-residual $G^{\mathfrak{r}}$ de $G$ es un subgrupo solitario (subnormal solitario) de G. Entonces la formación $\mathfrak{F}$ es cerrada para extensiones y subgrupos (normales).

Demostración. Probaremos primero que $\mathfrak{F}$ es cerrada para extensiones. Sea $G$ un grupo con un subgrupo normal $N$ tal que $G / N$ y $N$ pertenecen a $\mathfrak{F}$. Entonces $(G \times N)^{\mathscr{\mho}}=G^{\mathscr{F}} \times 1$ es un subgrupo (subnormal) solitario de $G \times N$. Pero como $G / N \in \mathfrak{F}, G^{\mathscr{Y}} \leqslant N$ y así $1 \times G^{\mathscr{F}}$ es un subgrupo normal de $G \times N$ isomorfo a $G^{\widetilde{r}} \times 1$. Dado que este es un subgrupo solitario de $G$, obtenemos que $G^{\widetilde{F}}=1$, es decir, $G \in \mathfrak{F}$. De ahí se sigue que $\mathfrak{F}$ es cerrada para extensiones.

Ahora demostramos que $\mathfrak{F}$ es cerrada para subgrupos (normales). Sea $H$ un subgrupo (normal) de $G \in \mathfrak{F}$. Entonces $(G \times H)^{\mathscr{\mho}}=1 \times H^{\mathscr{\mho}}$, pero $H^{\widetilde{r}} \times 1$ es un subgrupo (normal) de $G \times H$ isomorfo al subgrupo solitario (subnormal solitario) $1 \times H^{\widetilde{r}}$. Esto implica que $H^{\widetilde{\mho}}=1$, es decir, $H \in \mathfrak{F}$. En consecuencia, $\mathfrak{F}$ es cerrada para subgrupos (normales).

Podemos probar el resultado dual del teorema 4.7 para subgrupos solitarios para cocientes.

Teorema 4.9 ([ERL, Theorem 3.8]). Sea $\mathfrak{F}$ una clase de Fitting no vacía cerrada para extensiones y cocientes. Entonces el $\mathfrak{F}$-radical $G_{\mathfrak{r}}$ es solitario para cocientes en $G$.

Demostración. Supongamos que $N$ es un subgrupo normal de $G$ tal que $G / G_{\mathfrak{\gamma}}$ es isomorfo a $G / N$. Como $\mathfrak{F}$ es cerrada para extensiones, tenemos que $\left(G / G_{\mathfrak{F}}\right)_{\mathfrak{F}}=1$ por el teorema 1.32 (página 22). Por otra parte, $G_{\mathfrak{F}} N / N \cong G_{\mathfrak{F}} /\left(N \cap G_{\mathfrak{F}}\right) \in \mathfrak{F}$ porque $\mathfrak{F}$ es cerrada para cocientes. Pero como $G / N$ es isomorfo a $G / G_{\tilde{\mho}}, G / N$ no puede tener subgrupos normales no triviales en $\mathfrak{F}$. Resulta que $G_{\mathfrak{F}}=G_{\mathfrak{F}} \cap N$, es decir, $G_{\mathfrak{F}} \leqslant N$ y, por consideraciones de orden, concluimos que $G_{\tilde{F}}=N$.

La versión dual del teorema 4.8 para subgrupos solitarios para cocientes se da para clases de Fitting no vacías $\mathfrak{F}$ que satisfacen que $(G \times H)_{\mathfrak{\mho}}=G_{\mathfrak{\gamma}} \times H_{\mathfrak{\mho}}$ para cada dos grupos $G$ y $H$. Estas clases de Fitting se conocen como clases de Lockett. Un estudio detallado de las clases de Lockett aparece en [DH92, Chapter X, Section 1]. 
Teorema 4.10 ([ERL, Theorem 3.9]). Supongamos que $\mathfrak{F}$ es una clase de Lockett tal que, para cada grupo $G$, el $\mathfrak{F}$-radical es un subgrupo solitario para cocientes de G. Entonces I es cerrada para extensiones y cocientes.

Demostración. Probaremos primero que $\mathfrak{F}$ es cerrada para extensiones. Sea $G$ un grupo con un subgrupo normal $N$ tal que $G / N$ y $N$ pertenecen a $\mathfrak{F}$. Entonces $(G \times(G / N))_{\mathfrak{F}}=G_{\mathfrak{F}} \times(G / N)$ es un subgrupo solitario para cocientes de $G$ y $(G \times(G / N)) /\left(G_{\mathscr{\gamma}} \times(G / N)\right)$ es isomorfo a $G / G_{\mathfrak{F}}$. Como $N \in \mathfrak{F}, N \leqslant G_{\mathfrak{F}}$. Entonces el subgrupo normal $G \times\left(G_{\mathfrak{\gamma}} / N\right)$ satisface que $(G \times(G / N)) /\left(G \times\left(G_{\mathfrak{\gamma}} / N\right)\right)$ es isomorfo a $G / G_{\mathfrak{\gamma}}$. Como $G_{\mathfrak{\gamma}} \times(G / N)$ es solitario para cocientes, obtenemos que $G=G_{\mathfrak{\mho}}$, es decir, $G \in \mathfrak{F}$. Concluimos que $\mathfrak{F}$ es cerrada para extensiones.

Vamos a demostrar ahora que $\mathfrak{F}$ es cerrada para cocientes. Sea $N$ un subgrupo normal de $G \in \mathfrak{F}$. Entonces $(G \times(G / N))_{\mathfrak{F}}=G \times(G / N)_{\mathfrak{F}}$. Sea $X / N=(G / N)_{\mathfrak{\mho}}$. Tenemos que $(G \times(G / N)) /\left(G \times(G / N)_{\mathfrak{r}}\right) \cong G / X$. Entonces $X \times(G / N)$ es otro subgrupo normal de $G \times(G / N)$ que da un cociente isomorfo a $G / X$. Como $G \times(G / N)_{\mathfrak{F}}$ es solitario para cocientes, obtenemos que $(G / N)_{\mathfrak{F}}=G / N$, es decir, $G / N \in \mathfrak{F}$. Así $\mathfrak{F}$ es cerrada para cocientes.

\subsection{Subgrupos solitarios no radicales}

El hecho de que los radicales para una clase de Fitting no vacía sean subgrupos solitarios subnormales y los residuales para una formación no vacía sean subgrupos solitarios para cocientes motiva la pregunta de si todos los subgrupos subnormales solitarios son radicales para clases de Fitting adecuadas o si todos los subgrupos solitarios para cocientes pueden ser considerados como residuales para formaciones adecuadas. Por ejemplo, en el caso de $p$-grupos abelianos para un primo $p$, los subgrupos solitarios para cocientes son exactamente los residuales para las formaciones $\mathfrak{F}^{k}$, donde $\mathfrak{F}$ es la formación de todos $p$-grupos abelianos elementales, como veremos en el teorema 4.13. Esto ha sido demostrado por Tărnăuceanu [Tăr12b, Tăr12a]. Notemos que, para un p-grupo $G, \Phi(G)$ es el menor subgrupo normal que da cociente p-grupo abeliano elemental por [Hup67, Kapitel III, Satz 3.14], con lo que $\Phi(G)=G^{\mathscr{F}}$.

Recordemos que si $G$ es un p-grupo, $\Omega_{n}(G)$ es el subgrupo de $G$ generado por todos los elementos de $G$ cuyo orden es un divisor de $p^{n}$. 
Teorema 4.11 ([Tăr12a, Theorem]). Sea Gun p-grupo abeliano y sea $H \leqslant G$. Si $H$ es un subgrupo solitario de $G$, entonces $H=\Omega_{n}(G)$, donde $p^{n}=\exp H$.

Demostración. Denotemos $p^{n}=\exp H$ y sea $x \in H$ un elemento de orden $p^{n}$. Entonces $H \leqslant \Omega_{n}(G)$. Supongamos que $H<\Omega_{n}(G)$. Como $\Omega_{n}(G)$ está generado por los elementos de orden divisor de $p^{n}$ en $G$, existe $y \in \Omega_{n}(G)$ con $y^{p^{n}}=1$ y $y \notin H$. Si $y$ no tiene orden $p^{n}$, podemos considerar $x y$, que es un elemento de orden $p^{n}$ de $G$ con $x y \notin H$, luego podemos suponer sin pérdida de generalidad que $y$ tiene orden $p^{n}$. Por un resultado muy conocido de grupos abelianos (véase, por ejemplo, [Sch94, Lemma 2.3.11]), existen subgrupos $K$ y $L$ de $G$ tales que $\Omega_{n}(G)=\langle x\rangle \times K=\langle y\rangle \times L$. Por la identidad de Dedekind, $H=\langle x\rangle \times(H \cap K)$. Como $L$ y $K$ son grupos abelianos isomorfos, existe un subgrupo $L_{1} \leqslant L$ isomorfo a $H \cap K$. Se sigue que $\langle y\rangle \times L_{1} \mathrm{y}\langle x\rangle \times(H \cap K)=H$ son isomorfos. Como $y \notin H$, esto contradice que $H$ es un subgrupo solitario de $G$. Entonces $H=\Omega_{n}(G)$, como deseábamos.

El resultado siguiente es el dual del teorema 4.11. Podría demostrarse también recurriendo a la autodualidad del retículo de subgrupos de un grupo abeliano finito mostrada por Baer [Bae37] (véase también [Sch94, Theorem 8.1.4]), tal como hace Tărnăuceanu en [Tăr12b, Tăr12a]. Presentaremos una demostración directa de este hecho. Recordemos que para un $p$-grupo $G$ y un entero $n \geqslant 0, \mho_{n}(G)=$ $\left\langle\left\{g^{p^{n}} \mid g \in G\right\}\right\rangle$. Necesitaremos el siguiente lema, cuya prueba es en parte dual de la de [Sch94, Lemma 2.3.11].

Lema 4.12. Sea $G$ un p-grupo abeliano para un primo $p$ y sean $X$ y $H$ dos subgrupos de $G$ con $G / X$ cíclico de orden el exponente de $G$ y $X H=G$. Entonces existe un subgrupo $C$ de $G$ con $X \cap C=1, X C=G$ y $C \leqslant H$.

Demostración. Razonamos por inducción sobre $|G|$. Supongamos primero que $H \neq G$. Entonces $H /(X \cap H) \cong H X / X$ es un cociente cíclico de orden máximo de $H$. Por lo tanto, existe $C \leqslant G$ tal que $C \leqslant H$, $(X \cap H) C=H$ y $X \cap C=1$. De este modo, $X C=X(X \cap H) C=X H=G$ y $C$ cumple las condiciones deseadas. Por lo tanto, podemos suponer que $H=G$. Será suficiente con demostrar que $X$ tiene un complemento en $G$. Supongamos ahora que existe un subgrupo $Z \leqslant G$ de modo que $|G: Z|=p$ y $Z X=G$. Razonamos como antes con $Z$ en vez de $H$ y concluimos que existe un complemento $C$ de $H$ con $C \leqslant Z$. De este modo, tenemos que $X \leqslant \Phi(G)$. Pero entonces $G / \Phi(G)$ es cíclico y, por 
el teorema de la base de Burnside [Hup67, Kapitel III, Burnsidescher Basissatz 3.15], $G$ es también cíclico, con lo que $X=1$ y $C=1$ cumple la condición deseada.

Teorema 4.13. Sea $G$ un p-grupo abeliano para un primo $p$ y sea $H \leqslant G$. Si $H$ es un subgrupo solitario para cocientes de $G$, entonces $H=\mho_{n}(G)$ para un cierto entero $n \geqslant 0$.

Demostración. Existe un entero $n$ de manera que $\mho_{n}(G) \leqslant H$, pero $\mho_{n-1}(G) \nless H$. Supongamos que $\mho_{n}(G)$ es un subgrupo propio de $H$. Así, $G / H$ es un cociente de $G / \mho_{n}(G)$, que tiene exponente $p^{n}$, mientras que $G / H$ no es un cociente de $G / \widetilde{\mho}_{n-1}(G)$. Se concluye que el exponente de $G / H$ es $p^{n}$. Existe un subgrupo $X$ de $G$ con $H \leqslant X$ y $G / X \cong C_{p^{n}}$. Además, $G$ tiene un cociente $G / Y$ con $H \nless Y$ y $G / Y \cong C_{p^{n}}$ : en otro caso, $H$ estaría contenido en todos los subgrupos que dan cociente $C_{p^{n}}$, con lo que estaría contenido en todos los subgrupos que dan cociente de exponente divisor de $p^{n} \mathrm{y}$, como la clase de los grupos abelianos de exponente divisor de $p^{n}$ es una formación, $H$ estaría contenido en el residual para esta clase, que es $\mho_{n}(G)$, en contra de nuestra hipótesis. Por el lema 4.12, existen subgrupos $K$ y $L$ de $G$ con $X \cap K=Y \cap L=\mho_{n}(G)$, $X K=Y L=G$. De este modo, $H=H \mho_{n}(G)=H(X \cap K)=X \cap(H K)$. Como $G / L \cong G / K$, existe un subgrupo $L_{1}$ de $G$ de manera que $L \leqslant L_{1}$ y $G / L_{1} \cong G / H K$. Notemos que $G /\left(Y \cap L_{1}\right)$ es isomorfo a un subgrupo de $(G / Y) \times\left(G / L_{1}\right)$. Además, como $G=Y L \leqslant Y L_{1}$, se tiene que $G=Y L_{1}$. Por otra parte,

$$
\left|G /\left(Y \cap L_{1}\right)\right|=\frac{|G|}{|Y|\left|L_{1}\right| /\left|Y L_{1}\right|}=\frac{|G|^{2}}{|Y|\left|L_{1}\right|}=\left|(G / Y) \times\left(G / L_{1}\right)\right| .
$$

Por tanto, se tiene que $G /\left(Y \cap L_{1}\right) \cong(G / Y) \times\left(G / L_{1}\right)$. Análogamente, se tiene que $G / H=G /(X \cap H K) \cong(G / X) \times(G / H K) \cong(G / Y) \times\left(G / L_{1}\right) \cong$ $G /\left(Y \cap L_{1}\right)$. Como $H$ es solitario para cocientes, se sigue que $H=Y \cap L_{1}$. Pero esto contradice que $H \nless Y$. Concluimos que $H=\mho_{n}(G)$.

Sin embargo, la conjetura que nos planteamos al principio de la sección no es cierta en general. La clave para mostrar esto es observar que la formación (respectivamente, la clase de Fitting) más pequeña que contiene el grupo diédrico de orden 8 , también contiene el grupo cuaternio de orden 8 y la formación (respectivamente, la clase de Fitting) más pequeña que contiene el grupo cuaternio de orden 8 contiene 
el grupo de diédrico de orden 8. Por completitud, damos pruebas de estos hechos.

Lema 4.14 ([ERL, Lemma 4.10]). La clase de Fitting más pequeña que contiene el grupo cuaternio $Q_{8}$ de orden 8 coincide con la clase de Fitting más pequeña que contiene el grupo diédrico $D_{8}$ de orden 8 .

Demostración. Esto se deduce del hecho bien conocido que el grupo extraespecial de orden 32 , que es un producto central de dos copias de $D_{8}$, es isomorfo a un producto central de dos copias de $Q_{8}$ (véase [DH92, Chapter A, Lemma 20.4]).

Lema 4.15 ([ERL, Lemma 4.11]). La formación más pequeña que contiene el grupo cuaternio $Q_{8}$ de orden 8 coincide con la formación más pequeña que contiene el grupo diédrico $D_{8}$ de orden 8 .

Demostración. Sea

$$
G=\left\langle a, b, c \mid a^{4}=b^{4}=c^{2}=1, b^{a}=b c, c^{a}=c, c^{b}=c\right\rangle .
$$

Entonces $G$ posee cuatro subgrupos normales $N_{1}=\left\langle a^{2}, b^{2}\right\rangle, N_{2}=$ $\left\langle c a^{2}, b^{2}\right\rangle, N_{3}=\left\langle c b^{2}, a^{2}\right\rangle$ y $N_{4}=\left\langle c a^{2}, a^{2} b^{2}\right\rangle$ de manera que $N_{1} \cap N_{2} \cap N_{3}=1$, $G / N_{i} \cong D_{8}$ para $i \in\{1,2,3\}$ y $G / N_{4} \cong Q_{8}$. Esto prueba que $Q_{8}$ pertenece a la formación mas pequeña a la que pertenece $D_{8}$.

Ahora sea $H=\left\langle a, b, c \mid a^{4}=c^{4}=1, a^{2}=b^{2}, b^{a}=b^{3}, c^{a}=c^{3}, c^{b}=c\right\rangle$. Entonces $H$ tiene tres subgrupos normales $T_{1}=\langle c\rangle, T_{2}=\left\langle c a^{2}, c^{2}\right\rangle$, $T_{3}=\left\langle b^{3}, a^{2} c^{2}\right\rangle$ de manera que $G / T_{1} \cong G / T_{2} \cong G / T_{3} \cong Q_{8}$ y $T_{1} \cap T_{2} \cap T_{3}=1$ y un subgrupo normal $T_{4}=\langle b\rangle$ tal que $G / T_{4} \cong D_{8}$. Esto prueba que $D_{8}$ pertenece a la formación más pequeña que contiene $Q_{8}$.

Ejemplo 4.16 ([ERL, Example 4.12]). El grupo cuasidiédrico $G=\langle a, b, c|$ $\left.a^{4}=b^{2}=1, c^{2}=a^{2}, b^{a}=b a^{2}, c^{a}=c a^{2}, c^{b}=c a^{2}\right\rangle$ de orden 16 tiene subgrupos solitarios $\langle a, c\rangle \cong Q_{8} \mathrm{y}\langle b, c\rangle \cong D_{8}$. Por lo tanto, ninguno de ellos puede ser el radical para una clase de Fitting.

Ejemplo 4.17 ([ERL, Example 4.13]). Sea $G=\langle a, b| a^{4}=b^{4}=1, b^{a}=$ $\left.b^{3}\right\rangle$. Como $G$ tiene dos subgrupos solitarios para cocientes $A=\left\langle a^{2}\right\rangle$ y $B=\left\langle b^{2} a^{2}\right\rangle$, se tiene que $G / A \cong D_{8}$ y $G / B \cong Q_{8}$. Por lo tanto, ninguno de estos subgrupos puede ser el residual para una formación. 



\section{Capítulo 5}

\section{Grupos cuyos subgrupos minimales son solitarios}

Una de las líneas más fructíferas en la investigación en la teoría abstracta de grupos durante los últimos años ha sido el estudio de los grupos en los que los miembros de una determinada familia de subgrupos satisfacen una cierta propiedad de inmersión de subgrupos. La familia de los subgrupos de orden primo (también llamados subgrupos minimales) y la de los subgrupos maximales han atraído el interés de muchos matemáticos. Por ejemplo, un resultado bien conocido de Itô ([Itô55], véase [Hup67, Kapitel III, Satz 5.3]) afirma que un grupo de orden impar con todos los subgrupos minimales centrales es nilpotente.

El principal objetivo de este capítulo es el estudio de los grupos en los que todos los subgrupos minimales son solitarios. Estos son los grupos $G$ con un único subgrupo de orden $p$ para cada primo $p$ divisor de $|G|$. Los resultados de este capítulo forman parte del trabajo [ERL16].

\subsection{Grupos cuaternios generalizados}

El punto de partida de nuestra investigación de los grupos que poseen un único subgrupo de orden $p$ para cada primo $p$ divisor del orden del grupo es el siguiente bien conocido caso particular.

Teorema 5.1 ([Hup67, Kapitel III, Satz 8.2]). Sea p un primo. Supongamos que el p-grupo $G$ tiene un único subgrupo de orden $p$. 
1. Si $p>2$, entonces $G$ es cíclico.

2. Sip $=2$, entonces G es cíclico o isomorfo al grupo cuaternio generalizado $Q_{2^{s}}$ para un $s \geqslant 3$, con presentación

$$
Q_{2^{s}}=\left\langle x, y \mid x^{2^{s-1}}=1, x^{2^{s-2}}=y^{2}, x^{y}=x^{-1}\right\rangle .
$$

El teorema 5.1 muestra el interés del grupo cuaternio generalizado de orden $2^{s}$, para un cierto $s$, en el ámbito de nuestra clasificación. Los siguientes lemas presentan algunas propiedades conocidas de los grupos cuaternios generalizados. Se pueden encontrar, por ejemplo, en el trabajo de Conrad [Con13]. Damos sus demostraciones para la comodidad del lector.

Lema 5.2. Sea S un subgrupo no cíclico de un 2-grupo cuaternio generalizado $Q$. Entonces $S$ es cuaternio generalizado.

Demostración. Como $S$ no es cíclico, $S$ no está contenido en $\langle x\rangle$. Por lo tanto, existe un elemento $x^{u} y \in S \backslash\langle x\rangle$. Por otra parte, $|S: S \cap\langle x\rangle|=$ $|S\langle x\rangle:\langle x\rangle| \leqslant 2$. Sea $S \cap\langle x\rangle=\left\langle x^{2^{r}}\right\rangle$. Está claro que $S=\left\langle x^{2^{r}}, x^{u} y\right\rangle$ es un grupo cuaternio generalizado de orden $2^{s-r}$ si $s-r \geqslant 3$; en otro caso, $\left|\left\langle x^{2^{r}}\right\rangle\right| \leqslant 2$, con lo que $S$ sería cíclico, en contra de nuestra hipótesis.

Lema 5.3. El grupo cuaternio generalizado $Q=Q_{2^{s}}$ con presentación (5.1) para $s \geqslant 4$ no posee automorfismos de orden primo impar.

Demostración. Notemos que, por el teorema de la base de Burnside [Hup67, Kapitel III, Burnsidescher Basissatz 3.15], $Q$ posee exactamente tres subgrupos maximales, tantos como el grupo abeliano $C_{2} \times C_{2}$. Si $s \geqslant 4$, los subgrupos maximales $\left\langle x^{2}, y\right\rangle$ y $\left\langle x^{2}, x y\right\rangle$, por el lema 5.2, son isomorfos al grupo cuaternio generalizado $Q_{2^{s-1}}$. Por lo tanto, el subgrupo maximal cíclico $\langle x\rangle$ es un subgrupo solitario de $Q$. En particular, $\langle x\rangle \cong C_{2^{s-1}}$ es un subgrupo característico de $Q$. Sea $\alpha$ un automorfismo de $Q$ de orden primo impar. Entonces $\alpha$ puede ser considerado como un automorfismo de $\langle x\rangle$. Como el grupo de automorfismos de $C_{2^{s-1}}$ tiene orden $2^{s-2}$, por [Hup67, Kapitel I, Satz 4.6] resulta que $\alpha$ centraliza $\langle x\rangle$. Por otra parte, $\alpha$ puede ser considerado como un automorfismo de $Q /\langle x\rangle \cong C_{2}$. Resulta que $\alpha$ centraliza $Q /\langle x\rangle$. Por [DH92, Chapter A, Proposition 12.3], $\alpha$ centraliza a $Q$. Esta contradicción completa la demostración del lema. 
Lema 5.4. Si $N$ es un subgrupo normal no cíclico de un 2-grupo cuaternio generalizado $Q$, entonces $|Q: N| \leqslant 2$.

Demostración. Como $N \nless\langle x\rangle$, existe un elemento $x^{u} y \in N \backslash\langle x\rangle$. Por lo tanto,

$$
\left(x^{u} y\right)^{-1}\left(x^{u} y\right)^{x}=y^{-1} x^{-u} x^{-1} x^{u} y x=x^{2} \in N .
$$

De aquí resulta que $\left\langle x^{2}\right\rangle$ es un subgrupo propio de $N$ y $\left|Q:\left\langle x^{2}\right\rangle\right|=4$. Se sigue que $|Q: N| \leqslant 2$.

Lema 5.5. Si A/B es una sección abeliana elemental no cíclica de un 2-grupo cuaternio generalizado, entonces $A / B \cong C_{2} \times C_{2}$.

Demostración. Sea $A / B$ una sección no cíclica de $Q$. Entonces $A$ no puede ser cíclico. Por el lema 5.2, $A$ es cuaternio generalizado. Si $A / B$ es abeliano elemental, el subgrupo de Frattini $\Phi(A)$ de $A$ está contenido en $B$. Pero como $A$ está generado por dos elementos, $|A / \Phi(A)|=4$ y así $|A / B| \leqslant 4$. Como $|A / B|>2$, concluimos que $|A / B|=4$ y así $A / B \cong C_{2} \times C_{2}$.

\subsection{Enunciado del teorema}

Como se ve en el teorema 5.1, los grupos cuaternios generalizados tienen una gran importancia en la clasificación de los grupos cuyos subgrupos de orden primo son solitarios. Las siguientes construcciones de grupos también tendrán un papel importante en esta clasificación.

Definición 5.6. Sea $s$ un número natural con $s \geqslant 2$.

1. $V(s)=\left[Q_{8}\right] C_{3^{s}}$, donde $s \geqslant 2$ y el generador $a$ de $C_{3^{s}}$ actúa sobre $Q_{8}=\left\langle x, y \mid x^{4}=1, y^{2}=x^{2}, x^{y}=x^{-1}\right\rangle$ mediante $x^{a}=y, y^{a}=x y$.

2. $W(s)=\langle a, x, y| a^{3^{s}}=x^{8}=1, y^{2}=x^{4}, x^{y}=x^{-1},\left(x^{2}\right)^{a}=y, y^{a}=$ $\left.x^{2} y, a^{x^{3} y}=a^{-1}\right\rangle$.

Notemos que el grupo W(s) tiene un 2-subgrupo de Sylow isomorfo a un grupo cuaternio generalizado de orden 16 y tiene un subgrupo normal de índice 2 isomorfo a $V(s)$.

Ya estamos en condiciones de poder enunciar el primer resultado principal de este capítulo, que es el siguiente. 
Teorema 5.7 ([ERL16, Theorem 1.3]). Un grupo G tiene todos sus subgrupos de orden primo solitarios si, y solo si, G es un grupo de uno de los siguientes tipos:

1. $G=[L] H$, donde $H$ y $L$ son grupos cíclicos de órdenes coprimos, $|L|$ es impar y todo elemento de orden primo de H centraliza a L.

2. $G=[L](H \times Q)$, donde $H$ y L son grupos cíclicos de órdenes coprimos e impares, $Q$ es un 2-grupo cuaternio generalizado, todo elemento de orden primo de $H$ centraliza a $L$ y $Q^{\prime}$ centraliza a $L$ (en particular, $\left.\left|Q / C_{Q}(L)\right| \leqslant 4\right)$.

3. $G=[L](H \times V(s))$, donde $[Q] C \cong V(s)$, con $L$ y $H\{2,3\}^{\prime}$-grupos cíclicos de órdenes coprimos, $Q \cong Q_{8}, C \cong C_{3^{s}}$, $Q$ centraliza a $L$ y todos los elementos de orden primo de $H$ y $C$ centralizan a $L$.

4. $G=[L](H \times W(s))$, donde $L$ y $H$ son $\{2,3\}^{\prime}$-grupos cíclicos de órdenes coprimos, los elementos de orden primo de $H$ centralizan a $L$ y el subgrupo maximal $E C$ de $W(s)$ centraliza a $L\left(E=\left\langle x^{2}, y\right\rangle \cong Q_{8} y\right.$ $C=\langle a\rangle \cong C_{3^{s}}$.

Aquí $[V] X$ denota el producto semidirecto de un subgrupo normal $X$ que actúa sobre $V$.

\subsection{Demostración de los resultados}

Para demostrar el teorema 5.7 necesitamos unos resultados cuya prueba hemos encontrado en unas notas de Schmidt [Sch09]. Su demostración depende de los teoremas 1.39 y 1.40 (página 23).

Proposición 5.8 ([Sch09, Proposition 5]). Sea p un primo impar. Supongamos que $G$ tiene un único subgrupo de orden $p$. Entonces, o bien $G$ es p-nilpotente o bien $\mathrm{O}_{p}(G) \cap \mathrm{Z}(G)=1$. En particular, $\mathrm{C}_{G}\left(\Omega_{1}\left(\mathrm{O}_{p}(G)\right)\right)$ es p-nilpotente.

Demostración. Por el teorema 5.1, el p-subgrupo de Sylow $P$ de $G$ es cíclico, ya que tiene un único subgrupo de orden $p$. Por el teorema 1.40 (página 23), $P$ se descompone como un producto directo de $P \cap N^{\prime}$ y $P \cap Z(N)$, donde $N=\mathrm{N}_{G}(P)$. Como un $p$-grupo cíclico es directamente indescomponible, se sigue que o bien $P \leqslant Z(N)$ o $P \cap Z(N)=1$. En 
el primer caso, el teorema 1.39 (página 23) nos muestra que $G$ es $p$ nilpotente. En el segundo caso, se tiene que $\mathrm{O}_{p}(G) \cap \mathrm{Z}(G)=P \cap Z(G) \leqslant$ $P \cap \mathrm{Z}(N)=1$. La afirmación final se sigue del hecho de que $\Omega_{1}\left(\mathrm{O}_{p}(G)\right)$, el único subgrupo de orden $p$, es central en $C\left(\Omega_{1}\left(\mathrm{O}_{p}(G)\right)\right)$, con lo que estamos en el primer caso.

El siguiente resultado es la clave para establecer la resolubilidad de un grupo con todos los subgrupos minimales solitarios.

Teorema 5.9 (Schmidt [Sch09, Theorem 7]). Sea $p$ un primo impar. Cada grupo G con un único subgrupo de orden p es una extensión de un subgrupo normal p-nilpotente con un único subgrupo de orden p por un cociente cíclico de orden divisor de $p-1$ que actúa sobre él fielmente. Recíprocamente, cada extensión de este tipo tiene un único subgrupo de orden $p$. En particular, cada uno de esos grupos es p-resoluble de p-longitud igual a 1.

Demostración. Esto es simplemente porque $\mathrm{C}_{G}\left(\Omega_{1}\left(\mathrm{O}_{p}(G)\right)\right)$ es un subgrupo normal $p$-nilpotente con un único subgrupo de orden $p$ por la proposición 5.8 y $G / C_{G}\left(\Omega_{1}\left(\mathrm{O}_{p}(G)\right)\right)$ es isomorfo a un subgrupo del grupo de automorfismos de un grupo de orden $p$, que es cíclico de orden $p-1$. Recíprocamente, también se tiene que la extensión normaliza $\Omega_{1}(P)$ para el $p$-subgrupo de Sylow cíclico $P$. La afirmación final es clara.

Los grupos en los que todos los $p$-factores principales para un primo $p$ son cíclicos y G-isomorfos cuando se consideran como G-módulos por conjugación tienen un papel importante en la demostración del teorema principal. Esta clase de grupos aparece cuando se analizan los grupos en los que la permutabilidad con los subgrupos de Sylow es transitiva, o, equivalentemente, todo subgrupo subnormal es permutable con los subgrupos de Sylow, los llamados PST-grupos, y constituyen una localización de esta clase. Usaremos la siguiente propiedad de estos grupos.

Teorema 5.10 (véase [BBERA10, Theorem 2.1.8]). Sea G un grupo con residual nilpotente L. Las siguientes afirmaciones son equivalentes:

1. Para cada primo $p$, los p-factores principales de G son cíclicos y Gisomorfos cuando se consideran como módulos para $G$ por conjugación. 
2. L es un subgrupo de Hall abeliano de orden impar de G sobre el que G actúa por conjugación como un grupo de automorfismos de potencias.

Ya podemos demostrar nuestro resultado principal.

Demostración del teorema 5.7. Es claro que los grupos de los tipos 1, 2, 3 y 4 tienen todos los subgrupos de orden primo solitarios.

Supongamos ahora que $G$ tiene todos los subgrupos de orden primo solitarios. Vamos a demostrar que $G$ es de uno de los tipos 1, 2, 3 o 4 . Para mayor comodidad del lector, descomponemos la prueba en pasos enunciados por separado.

Paso 1. Ges resoluble.

Por el teorema 5.9, tenemos que $G$ es $p$-resoluble para todo primo impar $p$. En particular, cada factor de composición de $G$ es $p$-resoluble para todo primo impar $p$. De ello se desprende que todo factor de composición de $G$ debe ser cíclico.

Paso 2. Todo subgrupo de $G$ tiene todos los subgrupos de orden primo solitarios.

Fijamos un sistema de Hall $\mathbb{H}$ en $G$, que existe por el paso 1 y la proposición 1.8 (página 17). Si $p$ es un primo y $\pi$ es un conjunto de primos, denotamos por $G_{p}$ un $p$-subgrupo de Sylow de $G$ en $\mathbb{H}$ y por $G_{\pi}$ un $\pi$-subgrupo de Hall de $G$ en $\mathbb{H}$.

Paso 3. Si p es un primo impar, entonces $G_{p}$ es cíclico. Por otra parte, $G_{2}$ es cíclico o cuaternio generalizado.

Esto es una consecuencia del teorema 5.1 y del paso 2.

Paso 4. Todos los factores principales de $G$ son cíclicos o isomorfos a $C_{2} \times C_{2}$.

Sea $p$ un primo impar. Si $G_{p}$ es cíclico, todos los $p$-factores principales de $G$ son cíclicos. Si $G_{2}$ es cíclico, todos los 2-factores principales de $G$ son cíclicos. Supongamos que $G_{2}$ no es cíclico. Entonces $G_{2}$ es cuaternio generalizado por el teorema 5.1. Dado un 2-factor principal $A / B$ de $G$, tenemos que $A / B$ es isomorfo a una sección abeliana elemental de $G_{2}$. Pero cada sección abeliana elemental de $G_{2}$ es un grupo cíclico o un grupo abeliano elemental de orden 4 por el Lema 5.5.

Paso 5. Si $|G|$ no es divisible por 3, entonces $G$ no tiene factores principales isomorfos a $C_{2} \times C_{2}$.

Supongamos que $A / B$ es un factor principal de $G$ isomorfo a $C_{2} \times$ $C_{2}$. Se tiene que $G / C_{G}(A / B)$ es isomorfo a un subgrupo del grupo de automorfismos de $C_{2} \times C_{2}$, que es isomorfo a $\mathrm{GL}_{2}(2) \cong \Sigma_{3}$. Si 3 no divide 
a $|G|, G / C_{G}(A / B)$ tiene orden no divisible por 3 y así es un 2-grupo. Pero esto es imposible por [DH92, Chapter A, Proposition 3.12].

Paso 6. Supongamos que $G_{2}$ es cíclico o que $G$ no tiene factores principales isomorfos a $C_{2} \times C_{2}$. Entonces $G$ es superresoluble.

Esto es una consecuencia inmediata del paso 4.

Paso 7. Si $G_{2}$ es cíclico o $G$ no tiene factores principales isomorfos a $C_{2} \times C_{2}$, entonces, para cada primo $p$, los p-factores principales de $G$ son $G$-isomorfos.

Por el paso 6, G es superresoluble. Supongamos que $p_{1}<p_{2}<$ $\cdots<p_{r}$ son los divisores primos de $|G|$. Entonces, por el teorema 1.10 (página 18), $G$ tiene una torre de Sylow

$$
1=P_{r} \leqslant P_{r-1} \leqslant \cdots \leqslant P_{1} \leqslant P_{0}=G
$$

donde $P_{i} \Vdash G$ para todo $i$ y $P_{i-1} / P_{i}$ es un $p_{i}$-subgrupo de Sylow de $G / P_{i}$ para $1 \leqslant i \leqslant r$. Por otra parte, $P_{i-1} / P_{i}=G_{p_{i}} P_{i} / P_{i}$ para $1 \leqslant i \leqslant r$. Podemos refinar (5.2) a una serie principal de $G$. Si $G_{p_{i}}=\left\langle a_{i}\right\rangle$, con $\left|G_{p_{i}}\right|=p_{i}^{s_{i}}$, entonces los factores principales de esta serie tendrán la forma $\left\langle a_{i}^{p_{i}^{j}}\right\rangle P_{i} /\left\langle a_{i}^{p_{i}^{j+1}}\right\rangle P_{i}$ para $1 \leqslant i \leqslant r$ y $0 \leqslant j \leqslant s_{i}-1$. Dado $g \in G, a_{i}^{g} P_{i}=$ $\left(a_{i} P_{i}\right)^{g P_{i}}=\left(a_{i} P_{i}\right)^{r_{g} P_{i}}=a_{i}^{r_{g}} P_{i}$ para un cierto $r_{g} \in \mathbb{Z}$. Por lo tanto, $\left(a_{i}^{p_{i}^{j}}\right)^{g} P_{i}=$ $\left(a_{i}^{p_{i}^{j}}\right)^{r_{g}} P_{i}$ para $0 \leqslant j \leqslant s_{i}-1$. En particular, $\left(a_{i}^{p_{i}^{j}}\right)^{g}\left\langle a_{i}^{p_{i}^{j+1}}\right\rangle P_{i}=\left(a_{i}^{p_{i}^{j}}\right)^{r_{g}}\left\langle a_{i}^{p_{i}^{j+1}}\right\rangle P_{i}$ y $g$ induce el mismo automorfismo en todos los $p$-factores principales en esta serie. Por el teorema de Jordan-Hölder [DH92, Chapter A, Theorem 3.2], todos los $p$-factores principales de $G$ son $G$-isomorfos.

Paso 8. $R=G_{\{2,3\}}$ es normal en $G$.

El grupo $G_{2^{\prime}}$ tiene todos sus subgrupos de orden primo solitarios y un 2-subgrupo de Sylow trivial. Por el paso $6, G_{2^{\prime}}$ es superresoluble. Como 3 es el divisor primo más pequeño del orden de $G_{2^{\prime}}$ si 3 divide $|G|$, tenemos que el $3^{\prime}$-subgrupo de Hall $R$ es normal en $G_{2^{\prime}}$ por el teorema 1.10. En particular, $G_{3}$ normaliza a $R$. Por otro lado, el grupo $G_{3^{\prime}}$ tiene todos sus subgrupos de orden primo solitarios y su orden no es divisible por 3 . Por lo tanto, $G_{3^{\prime}}$ es superresoluble por los pasos 5 y 6 . Así, su 2'-subgrupo de Hall $R$ es normal en $G_{3^{\prime}}$, por el teorema 1.10. En particular, el 2-subgrupo de Sylow $G_{2}$ de $G_{3^{\prime}}$ normaliza a $R$. Se concluye que $R$ es normal en $G$.

Paso 9. Si $G_{2}$ es cíclico o $G$ no tiene factores principales isomorfos a $C_{2} \times C_{2}$, entonces el residual nilpotente $L$ de G es un subgrupo de Hall cíclico de orden 
impar y $G$ induce un grupo de automorfismos de potencia en $L$.

Por el Teorema 5.10, el residual nilpotente $L$ de $G$ es un subgrupo de Hall abeliano de $G$ de orden impar. Como los $p$-subgrupos de Sylow de $L$ son cíclicos y el producto directo de subgrupos cíclicos de órdenes coprimos dos a dos es cíclico, se sigue el resultado.

Sea $\pi$ el conjunto de los números primos divisores del orden del residual nilpotente $L$ de $G$.

Paso 10. Si $G_{2}$ es cíclico, entonces $G$ es un grupo de tipo 1.

Sea $H=G_{\pi^{\prime}}$. Como $H \cong G / L, H$ es nilpotente. Ahora bien, $H$ tiene todos sus subgrupos de Sylow cíclicos. Por lo tanto, $H$ es cíclico. Como todos los subgrupos de orden primo de $H$ son solitarios en $G$ y tienen intersección trivial con $L$, deben centralizar a $L$. Por lo tanto, $G=[L] H$ es un grupo de tipo 1.

Paso 11. Si $G_{2}$ no es cíclico y $G$ no tiene factores principales isomorfos a $C_{2} \times C_{2}$, entonces $G$ es un grupo de tipo 2 .

Sea $D$ un $\pi^{\prime}$-subgrupo de Hall de $G$. Notemos que $D$ es nilpotente, ya que $D \cong G / L$. Sea $H$ un $2^{\prime}$-subgrupo de Hall de $D$. Como $H$ es un producto directo de grupos cíclicos, $H$ es cíclico. Entonces $D$ es un producto directo de $H$ y un 2-grupo cuaternio generalizado $Q$. Por otra parte, como todos los subgrupos de orden primo de $H$ son solitarios en $G$ y tienen intersección trivial con $L$, centralizan a $L$. Por otro lado, $Q / C_{Q}(L)$ es un grupo de automorfismos de $L$. Como este grupo de automorfismos es abeliano, obtenemos que $Q^{\prime} \leqslant \mathrm{C}_{Q}(L)$.

Paso 12. Supongamos que $G$ no es superresoluble. Entonces $T=G_{\{2,3\}}$ tiene un único factor principal de orden $4, \mathrm{O}_{2^{\prime}, 2}(T) / \mathrm{O}_{2^{\prime}}(T) \cong Q_{8}$, y $T / \mathrm{O}_{2^{\prime}, 2}(T)$ es isomorfo a $\mathrm{C}_{3}$ o $\Sigma_{3}$.

Sea $T=G_{\{2,3\}}$. Notemos que $T$ y $G / R$ son $T$-isomorfos y así los factores principales de $T$ se pueden identificar con los factores principales de $G$ sobre $R$.

Consideremos la serie principal

$$
1=T_{0}<T_{1}<T_{2}<\cdots<T_{m-1}<T_{m}=T
$$

de $T$, que podemos suponer, sin pérdida de generalidad, que contiene los términos $\mathrm{O}_{2^{\prime}, 2}(T)$ y $\mathrm{O}_{2^{\prime}}(T)$. Al realizar la intersección de los miembros de esta serie con $Q=G_{2}$, obtenemos una serie normal

$$
1=T_{0} \cap Q \leqslant T_{1} \cap Q \leqslant T_{2} \cap Q \leqslant \cdots \leqslant T_{m-1} \cap Q \leqslant T_{m} \cap Q=Q
$$


de $Q$, y que, al ser $Q$ un 2-subgrupo de Sylow y $T_{i} / T_{i+1}$ un 2-factor, $\left(T_{i} \cap Q\right) /\left(T_{i+1} \cap Q\right) \cong T_{i+1}\left(Q \cap T_{i}\right) / T_{i+1}=Q T_{i+1} / T_{i+1} \cap T_{i} / T_{i+1}=T_{i} / T_{i+1}$.

Supongamos que $\left(T_{d} \cap Q\right) /\left(T_{d-1} \cap Q\right)$ es un factor de orden 4 , con lo que la sección $\left(T_{d} \cap Q\right) /\left(T_{d-1} \cap Q\right)$ es isomorfa a $C_{2} \times C_{2}$, y que entre todos los factores de orden $4, d$ es el más grande posible. Por lo tanto, $T_{d} \cap Q$ es un subgrupo normal no cíclico de $Q$. Por el Lema 5.2, $T_{d} \cap Q$ es un grupo cuaternio generalizado. Como $\left|\left(T_{d} \cap Q\right) /\left(T_{d-1} \cap Q\right)\right|=4$, tenemos que $T_{d-1} \cap Q$ es cíclico por el Lema 5.4. Se sigue que (5.4) contiene un único factor $\left(T_{d} \cap Q\right) /\left(T_{d-1} \cap Q\right)$ de orden 4 . Consecuentemente, a la vista del isomorfismo (5.5), la serie (5.3) contiene un único factor principal $T_{d} / T_{d-1}$ de orden 4 . Por otra parte, por el Lema 5.4, tenemos que $\left|Q: T_{d} \cap Q\right| \leqslant 2$. Sea $U=C_{T}\left(T_{d} / T_{d-1}\right)$. Entonces $U$ es un subgrupo normal de $T$ y $T / U$ es isomorfo a un subgrupo del grupo simétrico $\Sigma_{3}$ de grado 3 que contiene el grupo alternado $A_{3}$ de grado 3 , ya que es isomorfo a un subgrupo del grupo de automorfismos de $C_{2} \times C_{2}$ con 2-radical trivial por [DH92, Chapter B, Proposition 3.12]. Todos los demás 2-factores principales de $T$ son centrales. Como $\mathrm{O}_{2^{\prime}, 2}(T)$ es la intersección de los centralizadores de los 2-factores principales de $G$ por [DH92, Chapter A, Theorem 13.8], obtenemos que $\mathrm{O}_{2^{\prime}, 2}(T)=U$. Sea $Y=\mathrm{O}_{2^{\prime}}(T)$. Como $T / U$ tiene un subgrupo de orden 3, el grupo $U / Y$ tiene un automorfismo de orden 3 (notemos que este subgrupo de orden 3 no centraliza el factor principal $T_{d} / T_{d-1}$ por [DH92, Chapter A, Proposition 12.3] y por ser los demás 2-factores principales de orden 2 y que $\left.T_{d} \leqslant U\right)$. Por el lema $5.3, U / Y \cong Q_{8}$.

Paso 13. Supongamos que $G$ no es superresoluble. Sea $T=G_{\{2,3\}}$ y $U=$ $\mathrm{O}_{2^{\prime}, 2}(T)$. Si $T / U \cong C_{3}$, entonces $T \cong V(s)$ para un cierto $s \geqslant 2$.

Supongamos primero que $T / U \cong C_{3}$. Sea $C=G_{3}=\langle a\rangle \cong C_{3^{s}}$. El elemento $a^{3}$ pertenece a $U$ y, por lo tanto, pertenece al 3-subgrupo de Sylow $Y=\mathrm{O}_{3}(T)$. En consecuencia, $Y=\Phi(C)$. Como $Y$ es un subgrupo normal de $T$, por [DH92, Chapter A, Theorem 9.2(d)], $Y \leqslant \Phi(T)$. Pero $T / Y$ es un grupo 3-nilpotente. Resulta que $T$ es un grupo 3-nilpotente, ya que la clase de todos los grupos 3-nilpotentes es cerrada bajo extensiones de Frattini (véase [Hup67, Kapitel VI, Hilfssatz 6.3]). Por lo tanto, el 2-subgrupo de Sylow $Q=G_{2}$ de $G$ es normal en $T$ y es complementado por el 3-subgrupo de Sylow cíclico $C \cong C_{3^{\text {s }}}$. Por consiguiente, $T \cong V(s)$.

Paso 14. Supongamos que $G$ no es superresoluble. Sea $T=G_{\{2,3\}}$ y $U=$ 
$\mathrm{O}_{2^{\prime}, 2}(T)$. Si $T / U \cong \Sigma_{3}$, entonces $T \cong W(s)$ para un cierto $s \geqslant 2$.

Supongamos ahora que $T / U \cong \Sigma_{3}$. Sea $M / U$ un 3 -subgrupo de Sylow de $T / U$. En este caso, el 2-subgrupo de Sylow $Q=G_{2}$ de $G$ es isomorfo a un grupo cuaternio generalizado de orden 16 . Supongamos que $Q=\left\langle x, y \mid x^{8}=1, y^{2}=x^{4}, x^{y}=x^{-1}\right\rangle$ y que $a$ es un generador del 3-subgrupo de Sylow de $C=G_{3}$; además, el subgrupo $\left\langle x^{4}\right\rangle$ de orden 2 es un subgrupo normal de $T$, ya que es solitario, contenido en $\Phi(Q)$ y así $\left\langle x^{4}\right\rangle \leqslant \Phi(T)$, por [DH92, Chapter A, Theorem 9.2(d)], y el elemento $a^{3}$ pertenece a $U$, por lo tanto, pertenece al único 3-subgrupo de Sylow de $Y=\mathrm{O}_{3}(M)$ de $U$, y así $Y \leqslant \Phi(T)$. Si $\left\langle x^{4}\right\rangle Y<\Phi(T)$, como $U$ está contenido en el residual superresoluble de $T$ y $U /\left\langle x^{4}\right\rangle Y$ es un factor principal de $T$ isomorfo a $C_{2} \times C_{2}$, concluimos que $\Phi(T)=U$, pero entonces $T$ sería superresoluble, ya que la clase de los grupos superresolubles es una formación saturada por [DH92, Chapter IV, Examples 3.4], en contra de la existencia de un factor principal de tipo $C_{2} \times C_{2}$. Se sigue que el residual superresoluble de $T / \Phi(T)$ coincide con $U / \Phi(T)$ y es un subgrupo normal abeliano elemental de orden 4 y, por lo tanto, está complementado por un subgrupo $D / \Phi(T) \cong \Sigma_{3}$ por [BBE06, Theorem 4.2.17] y todos los complementos son conjugados. Por la conjugación, podemos suponer que un 2-subgrupo de Sylow de $D / \Phi(T)$ está contenido en el 2-subgrupo de Sylow $Q \Phi(T) / \Phi(T)$ de $T / \Phi(T)$ y $a \in D$. Además, si $D_{2}$ es un 2-subgrupo de Sylow de $D$ de modo que $D_{2} \Phi(T) / \Phi(T)$ sea el 2-subgrupo de Sylow referido anteriormente, $D_{2} \Phi(T)=D_{2}\left\langle x^{4}\right\rangle Y$ y $Q \Phi(T)=Q\left\langle x^{4}\right\rangle Y=Q Y$. De este modo, $D_{2} \leqslant Q Y$. Se sigue que $D_{2}$ está contenido en un conjugado de $Q$ por [Hup67, Kapitel VI, Satz 4.6], podemos suponer que $D_{2} \leqslant Q$. Por lo tanto, existe un elemento $z \in(Q \cap D) \backslash U$ y $a^{z} \Phi(T)=a^{-1} \Phi(T)$, esto es, $a^{z} a \in \Phi(T)$. Podemos suponer que $E=\left\langle x^{2}, y\right\rangle$ es el 2-subgrupo de Sylow de $M$ y que $a$ actúa sobre $E$ mediante $\left(x^{2}\right)^{a}=y, y^{a}=x^{2} y$. Como $z^{2} \in \Phi(T)$ y $z$ no es el elemento de orden 2 de $T$ porque $z \neq x^{4}$, $z$ es un elemento de orden 4 . Por otra parte, $z \notin E$. En consecuencia, tenemos que $z=x^{1+2 e} y$ para un cierto número entero $e$. Por otro lado, como $a^{z} a \in \Phi(T)$, este elemento centraliza $E$. Notemos que $y^{x}=x^{-1} y x=x^{-2} y$. Por lo tanto,

$$
\begin{aligned}
y & =y^{z^{-1} a z a}=y^{y^{-1} x^{-1-2 e} a x^{1+2 e} y a}=\left(x^{2(1+2 e)} y\right)^{a x^{1+2 e} y a} \\
& =\left(y^{(1+2 e)} x^{2} y\right)^{x^{1+2 e} y a}=\left(y^{2+2 e} y^{-1} x^{2} y\right)^{x^{1+2 e} y a}=\left(x^{4+4 e} x^{-2}\right)^{x^{1+e} y a} \\
& =\left(x^{2+4 e}\right)^{y a}=\left(x^{-2-4 e}\right)^{a}=y^{-1-2 e}
\end{aligned}
$$


lo que implica que $e \equiv 3(\bmod 4)$. Esto da la presentación de $W(s)$.

Paso 15. Supongamos que $G_{\{2,3\}} \cong V(s)$. Entonces $G$ es un grupo de tipo 3 .

Sean $Q=G_{2}$ y $C=G_{3}$, entonces $Q \cong Q_{8}, C \cong C_{3^{s}}$ y $Q C \cong V(s)$. Claramente, el 2'-subgrupo de Hall $G_{2^{\prime}}=R C$ es un grupo de tipo $1 \mathrm{y}$ así $R C=[L](H \times C)$, donde $H$ y $L$ son $\{2,3\}^{\prime}$-grupos cíclicos de órdenes primos entre sí, y todos los elementos de orden primo de $H C$ centralizan a $L$. En particular, todos los elementos de orden primo de $H$ y los elementos de orden primo de $C$ centralizan a $L$. Ahora bien, $Q C$ actúa sobre todos los $G$-factores principales de $R$, que son cíclicos. Por lo tanto, su subgrupo derivado $(Q C)^{\prime}=Q$ centraliza todos los $G$-factores principales de $R$. Por [DH92, Chapter A, Proposition 12.3], Q centraliza a $R$.

Paso 16. Supongamos que $G_{2,3} \cong W(s)$. Entonces $G$ es un grupo de tipo 4.

El 3'-subgrupo de Hall $G_{3^{\prime}}=R Q$ de $G$ es un grupo de tipo 2. Por lo tanto, $G_{3^{\prime}}=[L](H \times Q)$, donde $H$ y $L$ son $\{2,3\}^{\prime}$-grupos cíclicos de órdenes coprimos, y todos los elementos de orden primo de $H$ centralizan $L$. Por otra parte, todos los $G$-factores principales de $R$ son cíclicos. Por eso el subgrupo derivado $E C$ de $G_{\{2,3\}}$ centraliza todos los $G$-factores principales de R. Por [DH92, Chapter A, Proposition 12.4], $E C$ centraliza a $L$ y a $H$. Se concluye que $G$ es un grupo de tipo 4 .

\subsection{Observaciones finales}

Como los subgrupos solitarios son normales, se puede dar una demostración alternativa del paso 1 de la demostración del teorema 5.7 sin utilizar el teorema 5.9 ni los teoremas 1.39 y 1.40 de los que depende, usando el siguiente resultado de Gaschütz e Itô ([Itô55], véase también [Hup67, Kapitel IV, Satz 5.7]), tal y como se ha hecho en [ERL16].

Teorema 5.11. Un grupo G cuyos subgrupos minimales son normales es resoluble y su subgrupo derivado $G^{\prime}$ posee un 2-subgrupo de Sylow normal con cociente nilpotente.

También podríamos considerar el problema dual de la clasificación de los grupos con todos subgrupos maximales solitarios. Es claro que un grupo con todos los subgrupos maximales solitarios debe ser nilpotente, porque en este caso todos los subgrupos maximales son normales y podemos aplicar el teorema 1.16 (página 19). Los siguientes ejemplos 
fueron obtenidos con ayuda del sistema de álgebra computacional GAP [GAP15] y parecen indicar que la clasificación de los p-grupos con todos los subgrupos maximales solitarios, para un primo $p$, es un problema difícil.

Ejemplo 5.12. El grupo cuasidiédrico

$$
\mathrm{QD}_{16}=\left\langle a, b \mid a^{8}=b^{2}=1, a^{b}=a^{3}\right\rangle
$$

tiene tres subgrupos maximales $\left\langle a b^{-1}, a^{2}\right\rangle \cong Q_{8},\left\langle b, a^{2}\right\rangle \cong D_{8},\langle a\rangle \cong C_{8}$. Por lo tanto, todos ellos son solitarios.

Ejemplo 5.13. El grupo $G=C_{8} \times Q_{16}$ tiene siete subgrupos maximales, todos ellos solitarios en $G$.

Ejemplo 5.14. El grupo $G=\langle a, b, c, d, e, f| c=[b, a], d=[c, a], e=$ $[c, b], f=[d, a], a^{3}=c^{-3}=f, b^{3}=d^{3}=e^{3}=f^{3}=1,[d, a]=[e, a]=$ $[d, b]=[e, b]=f,[f, b]=[f, d]=[f, e]=[d, c]=[e, c]=[e, d]=1\rangle$, correspondiente al grupo $\operatorname{SmallGroup}(729,41)$ en la biblioteca de grupos de orden pequeño de GAP [GAP15], es otro ejemplo de un 3grupo 2-generado no cíclico en el que sus cuatro subgrupos maximales son solitarios.

No hemos sido capaces de generalizar el ejemplo 5.14 a primos cualesquiera.

También podemos considerar el resultado dual del teorema 5.7 obtenido al sustituir subgrupos minimales por subgrupos maximales y subgrupos solitarios por subgrupos solitarios para cocientes. El resultado es más simple.

Teorema 5.15. Supongamos que todos los subgrupos maximales de un grupo $G$ son solitarios para cocientes. Entonces $G$ es cíclico.

Demostración. En primer lugar, notemos que todos los subgrupos maximales de $G$ son normales. En particular, $G$ es nilpotente por [DH92, Chapter A, Theorem 8.3]. Sea $\pi(G)=\left\{p_{1}, \ldots, p_{r}\right\}$ y sea $P_{i}$ un $p_{i}$-subgrupo de Sylow de $G, 1 \leqslant i \leqslant r$. Por tanto, $G=P_{1} \times \cdots \times P_{r}$. Sea $M_{i}$ un subgrupo maximal de $P_{i}, 1 \leqslant i \leqslant r$. Entonces $T_{i}=P_{1} \times \cdots \times P_{i-1} \times M_{i} \times P_{i+1} \times \cdots \times P_{r}$ es un subgrupo maximal de $G$ de índice $p_{i}$. Como $T_{i}$ es solitario en $G$, se sigue que $T_{i}$ es el único subgrupo maximal de índice $p_{i}$ de $G$. Por lo tanto, $M_{i}$ debe ser el único subgrupo maximal de $P_{i}$. Se sigue que 
$P_{i}$ es cíclico por [Hup67, Kapitel III, Satz 3.16]. De este modo, G es un producto directo de grupos cíclicos de órdenes coprimos dos a dos, y así $G$ es también cíclico.

Los grupos con un único subgrupo de orden $p$ para cada primo $p$ divisor de su orden son grupos cuyos subgrupos minimales son solitarios para cocientes. Pero también hay otros ejemplos de grupos que satisfacen esta condición.

Ejemplo 5.16. Sea $G=\left\langle a, b \mid a^{4}=b^{4}=1, a^{b}=a^{-1}\right\rangle$. Entonces los subgrupos de $G$ de orden 2 son $N_{1}=\left\langle a^{2}\right\rangle, N_{2}=\left\langle b^{2}\right\rangle$ y $N_{3}=\left\langle a^{2} b^{2}\right\rangle$, todos ellos normales en $G$. Además, $G / N_{1} \cong C_{4} \times C_{2}, G / N_{2} \cong D_{8}, G / N_{3} \cong Q_{8}$, por tanto, todos los subgrupos minimales de $G$ son solitarios para cocientes. 



\section{Bibliografía}

[AF12] R. Atanasov y T.S. Foguel. Solitary solvable groups. Comm. Algebra, 40(6):2130-2139, 2012.

[Bae37] R. Baer. Dualism in abelian groups. Bull. Amer. Math. Soc., 43:121-124, 1937.

[BBE06] A. Ballester-Bolinches y L. M. Ezquerro. Classes of Finite Groups, tomo 584 de Mathematics and its Applications. Springer, New York, 2006.

[BBERA10] A. Ballester-Bolinches, R. Esteban-Romero y M. Asaad. Products of finite groups, tomo 53 de de Gruyter Expositions in Mathematics. Walter de Gruyter, Berlin, 2010.

[Bur11] W. Burnside. Theory of groups of finite order. Cambridge University Press, Cambridge, segunda edición, 1911.

[Con13] K. Conrad. Generalized quaternions. http://www. math. uconn. edu/ kconrad/blurbs/grouptheory/genquat . pdf, visitada 25/04/2015.

[DH78] K. Doerk y T. O. Hawkes. On the residual of a direct product. Arch. Math., 30:458-468, 1978.

[DH92] K. Doerk y T. Hawkes. Finite Soluble Groups, tomo 4 de De Gruyter Expositions in Mathematics. Walter de Gruyter, Berlin, New York, 1992.

[ERL] R. Esteban-Romero y O. Liriano. A note on solitary subgroups of finite groups. Comm. Algebra, en prensa. 
[ERL12] R. Esteban-Romero y O. Liriano. Subgrupos solitarios de grupos finitos. En IX Encuentro en Teoría de Grupos, página 10. Universidad Autónoma de Madrid, Madrid, junio 2012. Comunicación oral.

[ERL14] R. Esteban-Romero y O. Liriano. Subgrupos solitarios de grupos finitos. En $X$ Congreso Internacional de Investigación Científica. Universidad Autónoma de Santo Domingo, Santo Domingo, junio 2014. Comunicación oral.

[ERL16] R. Esteban-Romero y O. Liriano. Finite groups with all minimal subgroups solitary. J. Algebra Appl., 15(8):1650140 (9 pages), 2016.

[GAP15] The GAP Group. GAP - Groups, Algorithms, and Programming, Version 4.7.8, 2015. http: //www . gap-system.org.

[Gor80] D. Gorenstein. Finite Groups. Chelsea Pub. Co., New York, 1980.

[Hal28] P. Hall. A note on soluble groups. J. London Math. Soc., 3:98-105, 1928.

[Hal37a] P. Hall. A characteristic property of soluble groups. J. London Math. Soc., 12:198-200, 1937.

[Hal37b] P. Hall. On the Sylow systems of a soluble group. Proc. London Math. Soc. (2), 43:316-323, 1937.

[Hup67] B. Huppert. Endliche Gruppen I, tomo 134 de Grund. Math. Wiss. Springer Verlag, Berlin, Heidelberg, New York, 1967.

[Itô55] N. Itô. Über eine zur Frattini-Gruppe duale Bildung. Nagoya Math. J., 62:400-401, 1955.

[KL09] G. Kaplan y D. Levy. Solitary subgroups. Comm. Algebra, 37(6):1873-1883, 2009.

[LC11] Orieta Liriano Castro. Subgrupos solitarios de grupos finitos. Trabajo de investigación de tercer ciclo, Universitat Politècnica de València, junio 2011. 
[LC13] O. Liriano Castro. Subgrupos solitarios de grupos finitos. Charla en Seminari Predoc, Universitat de València.

[Lev14] D. Levy. Corrigendum to: "Solitary subgroups". Comm. Algebra, 42(4):1855, 2014.

[Ros78] J.S. Rose. A Course on Group Theory. Cambridge University Press, London, New York, Melbourne, 1978.

[Sch94] R. Schmidt. Subgroup lattices of groups, tomo 14 de De Gruyter Expositions in Mathematics. Walter de Gruyter, Berlin, 1994.

[Sch09] J. Schmidt. Finite groups with a unique subgroup of order $p$. URL http://www.ms.uky.edu/ jack/2009-07-08-UniqueSubgroup0fOrderP.pdf, visitada $25 / 04 / 2015$.

[Tăr12a] M. Tărnăuceanu. Erratum to: "Solitary quotients of finite groups". Cent. Eur. J. Math., 11(2):376-377, 2012.

[Tăr12b] M. Tărnăuceanu. Solitary quotients of finite groups. Cent. Eur. J. Math., 10(2):740-747, 2012.

[Thé93] J. Thévenaz. Most finite groups are p-nilpotent. Expo. Math., 11(4):359-363, 1993. 